\title{
Existence and Uniqueness of the Solution of Lorentz-Rössler Systems with Random Perturbations
}

\author{
Xiaoying Wang, ${ }^{1,2}$ Fei Jiang, ${ }^{3}$ and Junping Yin ${ }^{4}$ \\ ${ }^{1}$ School of Mathematics and Physics, North China Electric Power University, Beijing 102206, China \\ ${ }^{2}$ Institute of Applied Mathematics, Academy of Mathematics and Systems Science, Chinese Academy of Sciences, Beijing 100190, China \\ ${ }^{3}$ College of Mathematics and Computer Science, Fuzhou University, Fuzhou 350108, China \\ ${ }^{4}$ Institute of Applied Physics and Computational Mathematics, Beijing 100088, China
}

Correspondence should be addressed to Xiaoying Wang; wangxy022@126.com and Junping Yin; yinjp829829@126.com

Received 24 December 2012; Accepted 26 January 2013

Academic Editor: Jinhu Lü

Copyright (C) 2013 Xiaoying Wang et al. This is an open access article distributed under the Creative Commons Attribution License, which permits unrestricted use, distribution, and reproduction in any medium, provided the original work is properly cited.

We consider a new chaotic system based on merging two well-known systems (the Lorentz and Rössler systems). Meanwhile, taking into account the effect of environmental noise, we incorporate whit-enoise in each equation. We prove the existence, uniqueness, and the moments estimations of the Lorentz-Rössler systems. Numerical experiments show the applications of our systems and illustrate the results.

\section{Introduction}

The Lorentz system is a well-known model

$$
\begin{gathered}
\dot{x}_{1}=\sigma\left(x_{2}-x_{1}\right), \\
\dot{x}_{2}=r x_{1}-x_{2}-x_{1} x_{3}, \\
\dot{x}_{3}=x_{1} x_{2}-\beta x_{3} .
\end{gathered}
$$

This model was introduced in 1963 by Lorentz [1]. For the meaning of the Lorentz system the reader can refer to [2] (Chaos), [3] (laser), [4] (thermospheres), [5] (brushless DC motors), [6] (electric circuits), and [7] (chemical reactions). The original Rössler system only contains one quadratic nonlinear term $x_{1} x_{3}$

$$
\begin{gathered}
\dot{x}_{1}=x_{2}-x_{3}, \\
\dot{x}_{2}=x_{1}+a x_{2}, \\
\dot{x}_{3}=b+x_{3}\left(x_{1}-c\right) .
\end{gathered}
$$

The system (2) was introduced by Rössler [8]. This model has received increasing attention due to its great potential applications in secure communication [9-12], chemical reaction, biological systems, and so on [13].
Furthermore, the general chaotic systems have many applications, especially in complex genetic networks [14-17]. Of course, since the general chaotic systems are nonlinear and have stochastic noise terms, lots of mathematical experts pay still their attentions to the mathematical theory for these nonlinear chaotic systems [18-22] and so on.

In this paper, we consider a new model including the Lorentz system and the Rössler system, which has been slightly adjusted (the nonlinear terms of the Rössler systems become $\left.x_{1}\left(x_{3}-c\right)\right)$ :

$$
\begin{gathered}
\dot{x}_{1}=\sigma\left(x_{2}-x_{1}\right)-\gamma(t)\left(x_{2}-x_{3}\right), \\
\dot{x}_{2}=r x_{1}-x_{2}-\alpha_{1}(t) x_{1} x_{3}+x_{1}+a x_{2}, \\
\dot{x}_{3}=\alpha_{2}(t) x_{1} x_{2}-\beta x_{3}+b+\alpha_{3}(t) x_{1}\left(x_{3}-c\right) .
\end{gathered}
$$

Actually, if we take some especial coefficients, the system (3) would become the system (1) or (2). Therefore, the main properties of the Lorentz and Rössler systems can be included by this model. In Figure 1, we take that $\alpha_{3}(t)=0, b=0$, $\alpha_{1}(t)=\alpha_{2}(t)=1, a=-1$, and that our problem becomes the well-known Lorentz system with the initial value $X_{0}=$ $(8,5,30)$. 


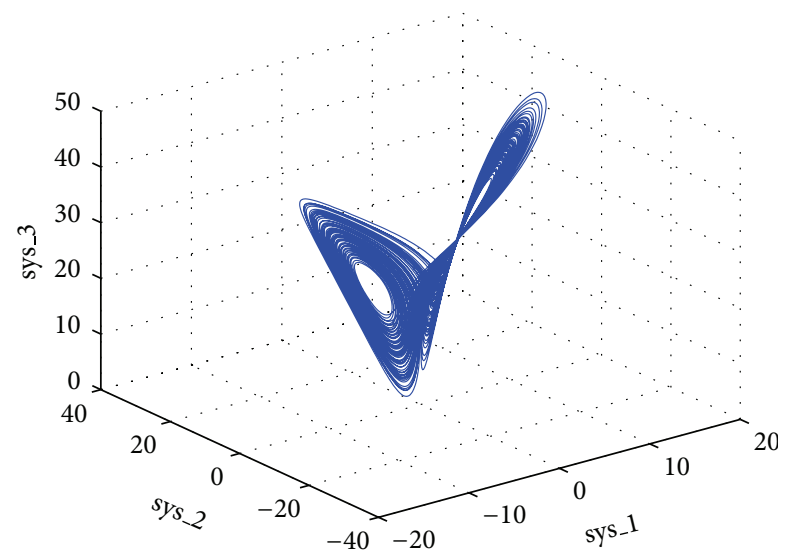

(a)
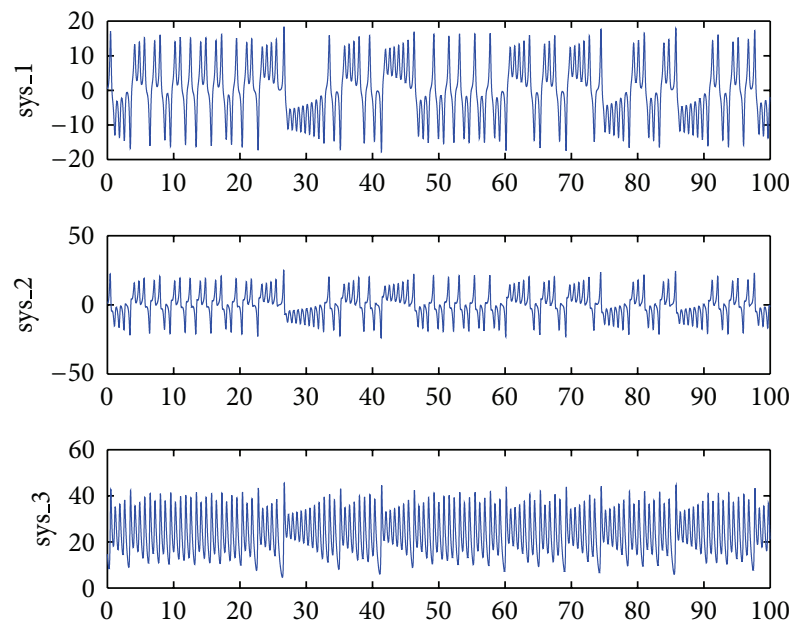

(b)

FIGURE 1: The attractor and the time series of the Lorentz system.

Let $\gamma(t)=1, \alpha_{1}(t)=\alpha_{2}(t)=0$, and $\sigma=0$, and take the correspondingly appropriate other parameters, then the track similar with the Rössler systems can be obtained.

If the coefficients of our problem are $\sigma=9, \gamma(t)=-1$, $r=27, a=b=c=0, \alpha_{1}(t)=\alpha_{2}(t)=2, \alpha_{3}(t)=1$, and $\beta=8 / 3$, then the system (3) becomes the following LorentzRössler system.

Remark 1. From the structure of system (3), it is obvious that there is a great diversification of attractors in the system inner with different parameters. Since the structure of our systems is more complex than the Lorentz system and Rössler system, especially, from Figure 3, the system (3) can be used in secure communications, to design the more complex hop-frequency communications time series, which make the communications content more secure. If we use the system (3) to design the hop-frequency time series in communications, it becomes much more difficult to disturb our communications than the time series of the well-known Lorentz system and Rössler system.

To obtain better applications about model (3), we must take into account the effect of environment noise,

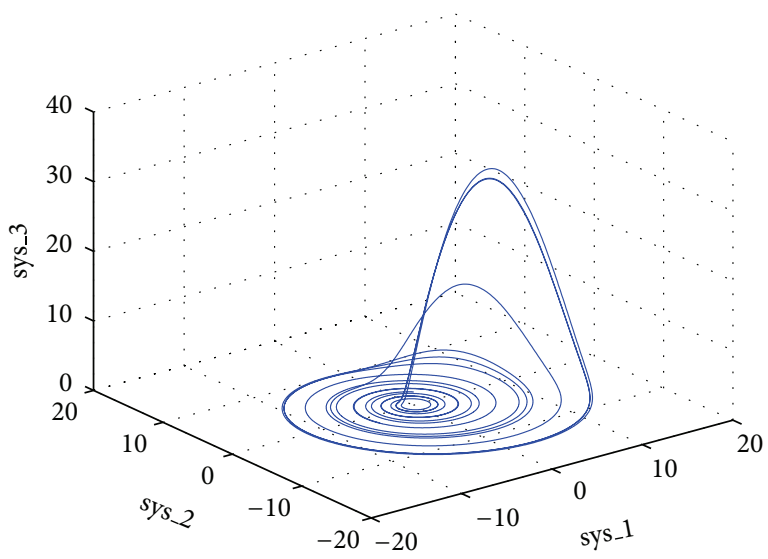

(a)
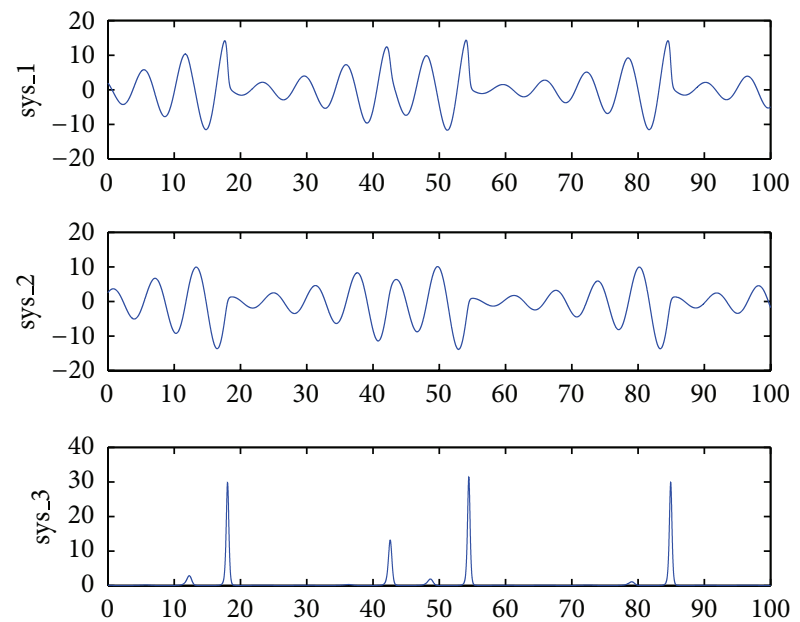

(b)

FIgURE 2: The behavior similar with the Rössler system (initial value $\left.X_{0}=(3,-4,2)\right)$.

especially in secure outer communications (complex electric circumstance), convulsed circuits communications, multilevel chemical reactions, and so on. Thus we incorporate white noise in each equation of system (3)

$$
\begin{aligned}
d x_{1}= & {\left[\sigma\left(x_{2}-x_{1}\right)-\gamma(t)\left(x_{2}-x_{3}\right)\right] d t } \\
& +\sum_{i=1}^{3} u_{1 i}\left(x_{1}, x_{2}, x_{3}\right) d B_{i}(t), \\
d x_{2}= & {\left[r x_{1}-x_{2}-\alpha_{1}(t) x_{1} x_{3}+x_{1}+a x_{2}\right] d t } \\
& +\sum_{i=1}^{3} u_{2 i}\left(x_{1}, x_{2}, x_{3}\right) d B_{i}(t), \\
d x_{3}= & {\left[\alpha_{2}(t) x_{1} x_{2}-\beta x_{3}+b+\alpha_{3}(t) x_{1}\left(x_{3}-c\right)\right] d t } \\
& +\sum_{i=1}^{3} u_{3 i}\left(x_{1}, x_{2}, x_{3}\right) d B_{i}(t),
\end{aligned}
$$




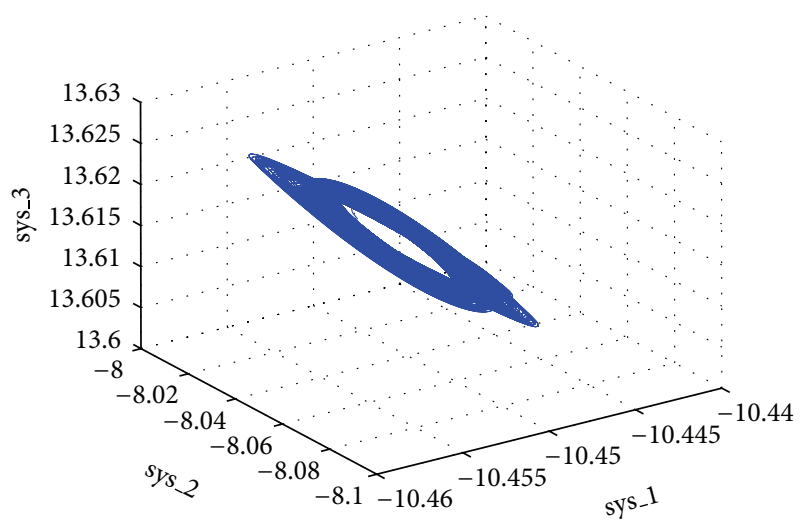

(a)
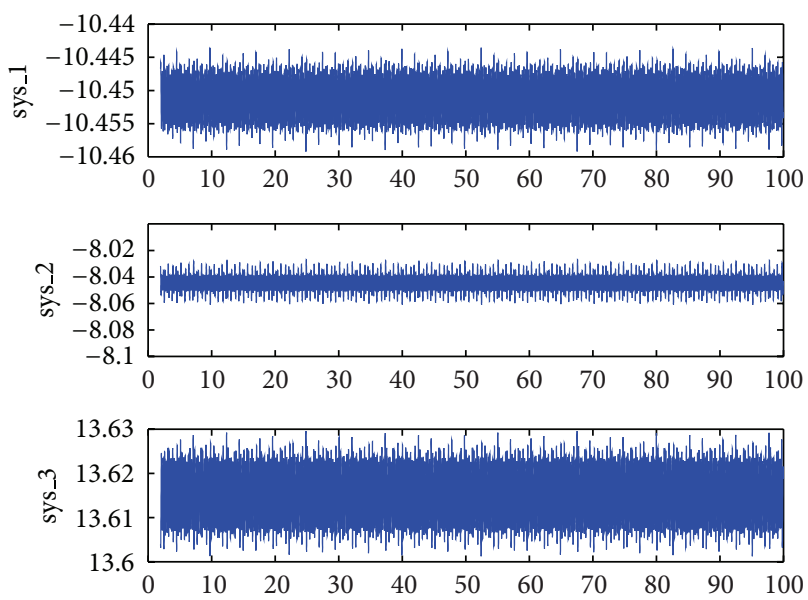

(b)

FIgURE 3: The Lorentz-Rössler combining system with initial value $X_{0}=(1,0.1,1)$.

where all the $u_{i j}$ represent the intensity of the noise at time $t$ and all the $B_{i}(t)$ are standard white noise; namely, each $B_{i}(t)$ is a Brownian motion defined on a complete probability space $(\Omega, \mathscr{F}, \mathbb{P})$. If we consider the Figures 1,2 , and 3 with environment noise, the respective stochastic system can be shown by Figures 4, 5, and 6, respectively.

In this paper, we consider that $\gamma(t)$ is not a constant and dependent on the third variable and the coefficient of the third interactive term $\alpha_{3}(t)$, let $\gamma(t)=\alpha_{3}(t) x_{3}(t)$. Throughout this paper, unless otherwise specified, we let $\left(\Omega, \mathscr{F},\left\{\mathscr{F}_{t}\right\}_{t \geq 0}, \mathbb{P}\right)$ be a complete probability with a filtration $\left\{\mathscr{F}_{t}\right\}_{t \geq 0}$ satisfying the usual conditions (i.e., it is right continuous and increasing while $\mathscr{F}_{0}$ contains all $\mathbb{P}$-null sets). $B(t):=\left(B_{1}(t), B_{2}(t), B_{3}(t)\right)^{T}$ denotes an 3-dimensional Brownian motion defined on this probability space.

The rest of this paper is arranged as follows. In Section 2, we introduce some fundamental conditions of our problem. In Section 3, the existence and uniqueness of the stochastic system (4) are established. Meanwhile, in our main results, the moments estimations of solutions are obtained. Section 4

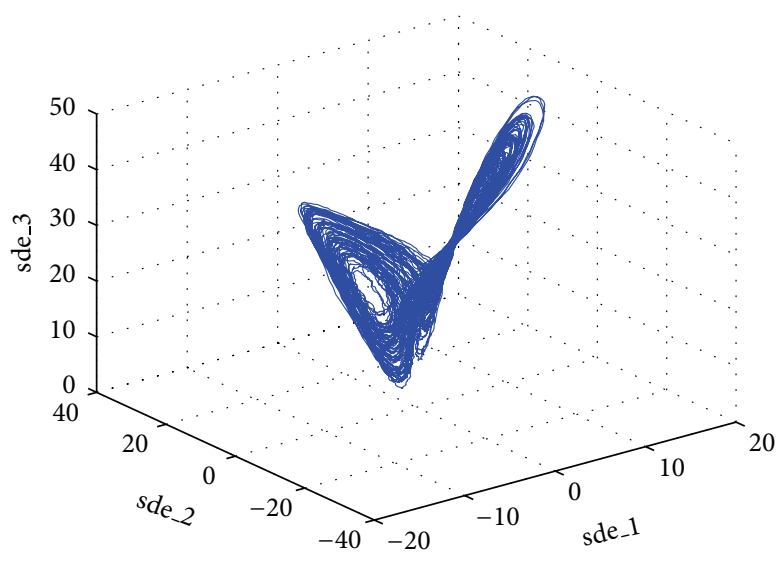

(a)
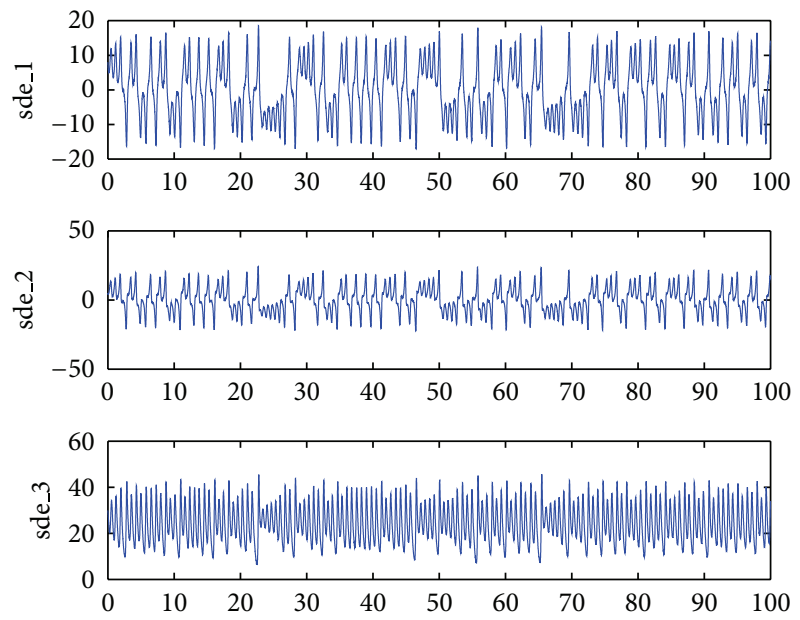

(b)

FIGURE 4: The attractor and the time series of the stochastic Lorentz system with a standard irrelated white noise $\left(u_{i i}=1, u_{i j, i \neq j}=1\right.$, $i, j=1,2,3)$.

shows some numerical simulations with inner random perturbations and outer random perturbations, which can support our results and exhibit diverse behaviors with different inner perturbations.

\section{Fundamental Assumptions and Notations}

We firstly split the system (4) into different parts and give some fundamental conditions. In this paper, we consider the generalized system (4) only forward in time $t \in[0, \infty)$. Let $X=\left(x_{1}, x_{2}, x_{3}\right) \in \mathbb{R}^{3}$. The Lorentz-Rössler system can be rewritten as

$$
\begin{gathered}
d X=-[A X+C(X)-F] d t+U(X, t) d B_{t}, \\
X(0)=X_{0}, \quad 0 \leq t \leq T<+\infty,
\end{gathered}
$$

where the initial condition $X_{0}=\left(x_{10}, x_{20}, x_{30}\right)^{T}$ is fixed point and independent of $\mathscr{F}_{t}$ for all $t>0$. The four parts of the drift 


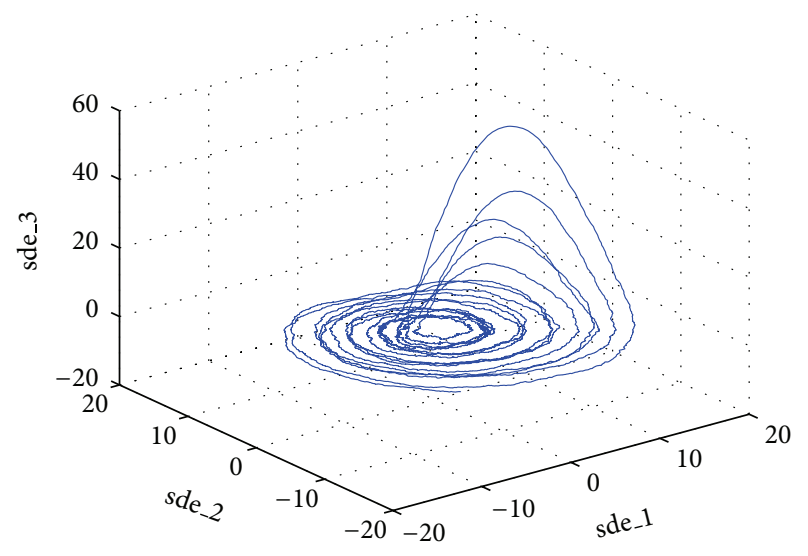

(a)
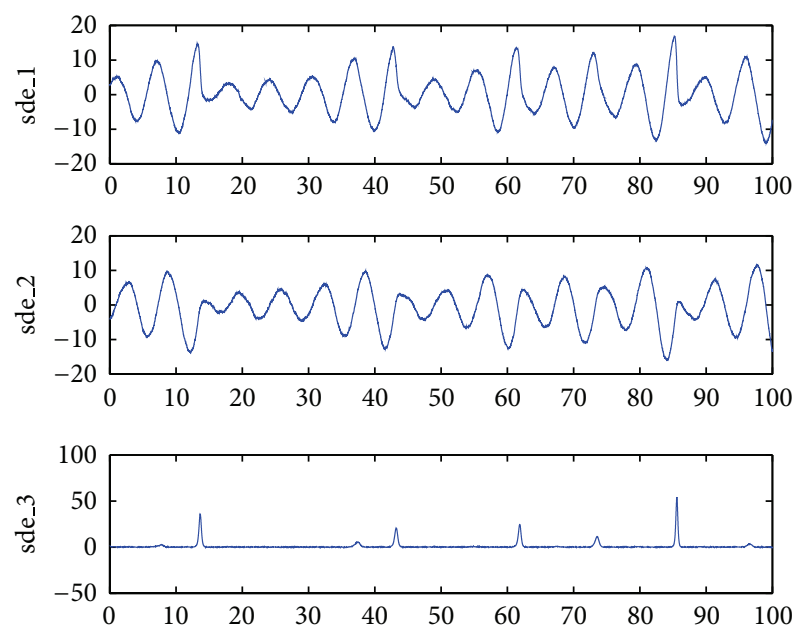

(b)

FIGURE 5: The behavior similar with the Rössler system (intensity of the noise $\left.u_{i i}=0.5, u_{i j, i \neq j}=0, i, j=1,2,3\right)$.

are given by

$$
\begin{gathered}
A=\left(\begin{array}{ccc}
\sigma & -\sigma & 0 \\
-(r+1) & 1-a & 0 \\
-\alpha_{3}(t) c & 0 & \beta
\end{array}\right) \\
C(X)=\left(\begin{array}{c}
-\alpha_{3}(t) x_{3} x_{2}+\alpha_{3}(t) x_{3}^{2} \\
\alpha_{1}(t) x_{1} x_{3} \\
-\alpha_{2}(t) x_{1} x_{2}-\alpha_{3}(t) x_{1} x_{3}
\end{array}\right) \\
F=\left(\begin{array}{l}
0 \\
0 \\
b
\end{array}\right), \quad U(X, t)=\left(\begin{array}{lll}
u_{11}(t) & u_{12}(t) & u_{13}(t) \\
u_{21}(t) & u_{22}(t) & u_{23}(t) \\
u_{31}(t) & u_{32}(t) & u_{33}(t)
\end{array}\right) .
\end{gathered}
$$

$U(X, t): \mathbb{R}^{3} \times[0, \infty) \rightarrow 3 \times 3$ matrices is a noise term.

For the sake of brevity, we introduce some notations.

(i) For any real matrix $R=\left[r_{i j}\right] \in \mathbb{R}^{d \times m}$, define $\|R\|_{2}^{2}=$ $\operatorname{trace}\left(R^{T} R\right)=\operatorname{trace}\left(R R^{T}\right)=\sum_{i, j} r_{i j}^{2}$.

(ii) For any $p \in \mathbb{N}$ is even, $X \in \mathbb{R}^{3},\|X\|_{2}^{p}=\left(x_{1}^{2}+x_{2}^{2}+\right.$ $\left.x_{3}^{2}\right)^{p / 2}$.

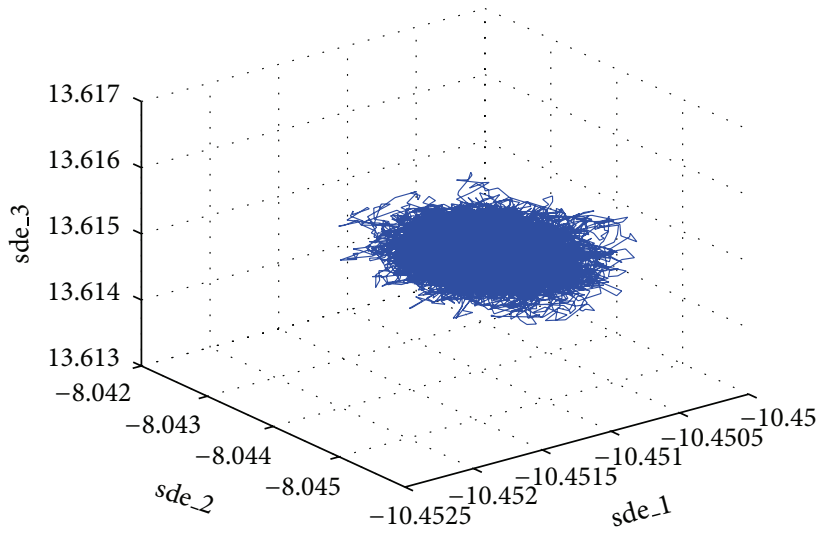

(a)
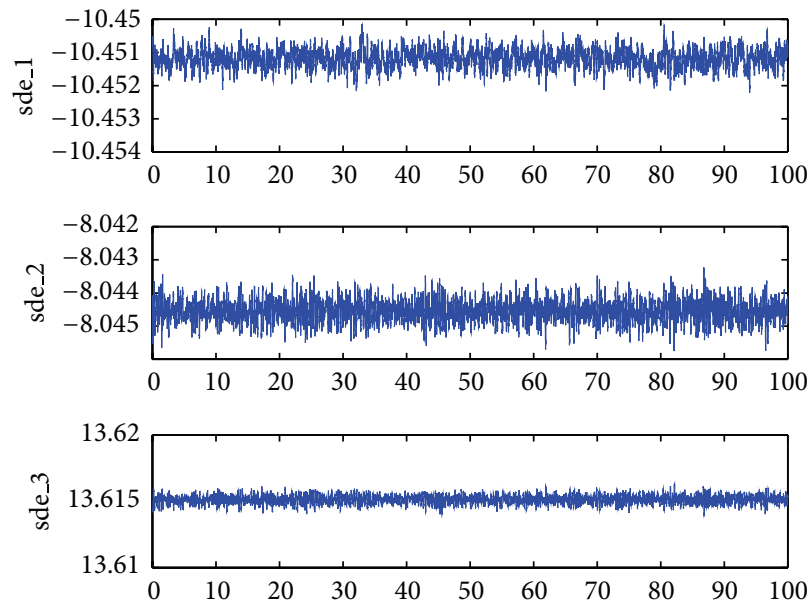

(b)

FIGURE 6: The Lorentz-Rössler combining system with the noise $u_{i i}=0.001, u_{i j, i \neq j}=0, i, j=1,2,3$.

(iii) For any two variables $X, Y \in \mathbb{R}^{3},(X, Y)$ denote the usual inner product.

(iv) $C_{i}(i=1,2, \ldots)$ denote the constants which are dependent on some parameters.

(A1) The noise term $U(X, t)$ satisfies a Lipschitz condition and a linear growth condition

$$
\|U(X, t)\|_{2}^{2}:=\operatorname{trace}\left(U(X, t) U^{T}(X, t)\right) \leq C_{1}\left(1+\|X\|_{2}^{2}\right) .
$$

The condition (A1) can be easily satisfied, for example, $U_{i}(X, t)=x_{i}(t) \sum_{j=1}^{3} \sigma_{i j}(t) d B_{j}(t)$, when all the $\sigma_{i j}(t)$ are bounded on $\mathbb{R}_{+}$.

For the other coefficients, we suppose the following conditions. 0 .

(A2) The matrix $A$ satisfies $(A X, X) \geq \lambda\|X\|_{2}^{2}$, where $\lambda>$

(A3) The constant $b$ and the coefficients $\alpha_{1}(t), \alpha_{2}(t)$, and $\alpha_{3}(t)$ are bounded. In addition, the interactive terms' coefficients satisfy $\alpha_{1}(t)=\alpha_{2}(t)+\alpha_{3}(t)$. 
Remark 2. If $U(X, t) \equiv 0$, our Lorentz-Rössler systems become a deterministic system. As for the deterministic equation, there are many methods to study. In this paper, we mainly consider the stochastic system. In any case, it is important to study the properties of the system in a weak noise environment.

\section{Main Results}

In this section, we will consider the stochastic equation (4) with initial value $X(0)=X_{0}=\left(x_{10}, x_{20}, x_{30}\right)^{T} \in \mathbb{R}^{3}$ and $U(X, t) \neq 0$. In order for a stochastic differential equation to have a unique global (i.e., no explosion in a finite time) solution for any given initial value, the terms of the equation are generally required to satisfy the linear growth condition and local Lipschitz condition. In (5), the terms $-A(t) X$, $F, U(X, t)$ satisfy these two conditions. However, the term $C(X, t)$ of (5) does not satisfy the linear growth condition, though they are locally Lipschitz continuous.

At first, we introduce the modified system. Let us study a modified system obtained by truncating the term, when it is too large. Some a priori moment estimates of the solution of the modified equation enable us to prove that the modified system converges to a solution of the original problem as the truncation level goes to infinity.

Lemma 3. Let $\chi_{N} \in C^{1}\left(\mathbb{R}^{3}, \mathbb{R}\right)$ with $\chi_{N}(X)=1$ for $\|X\|_{2} \leq N$ and $\chi_{N}(X)=0$ for $\|X\|_{2} \geq N+1 . C_{N}(X):=\chi_{N}(X) C(X)$, the modified system

$$
\begin{array}{r}
d X_{N}=-\left(A X_{N}+C_{N}\left(X_{N}\right)-F\right) d t+U\left(X_{N}\right) d B_{t}, \\
t \in[0, T], \quad X_{N}(0)=X_{0},
\end{array}
$$

where $X_{N}=\left(x_{N 1}, x_{N 2}, x_{N 3}\right)$ and $T$ is a finite time. And let the initial value be still independent of $\left\{\mathscr{F}_{t}\right\}_{t>0}$ and satisfy $E\left\|X_{0}\right\|_{2}^{2}<\infty$. Then the modified system (9) possesses a continuous almost sure unique solution that is $\left\{\mathscr{F}_{t}\right\}$ measurable.

Remark 4. $C_{N}\left(X_{N}\right)$ is bounded and satisfies a linear growth condition and a Lipschitz condition (it can be easily obtained by the insert-value technique and primary inequalities). All other coefficients obviously satisfy a linear growth as well as a Lipschitz condition. Since the truncation function $\chi_{N} \in$ $C^{1}\left(\mathbb{R}^{3}, \mathbb{R}\right)$, the modified nonlinear term $C_{N}\left(X_{N}\right)$ remains differentiable, and its derivative is continuous and has a compact support. Then the assertions of Lemma 3 follow by the usual existence and uniqueness theorem.

To get the uniform estimations of the system (9), we deal with the Itŏ derivative of the Lyapunov functions.

Lemma 5. Let (A2) and (A3) hold. Then one can get the estimation

$$
\begin{aligned}
d\left\|X_{N}\right\|_{2}^{p}= & -\frac{p \lambda}{2}\left\|X_{N}\right\|_{2}^{p} d t+\left\|X_{N}\right\|_{2}^{p-2} \frac{p b^{2}}{2 \lambda} d t \\
& +\frac{p}{2}(p-1)\left\|X_{N}\right\|_{2}^{p-2}\left\|U\left(X_{N}\right)\right\|_{2}^{2} d t
\end{aligned}
$$

$$
+p\left\|X_{N}\right\|_{2}^{p-2} X_{N}^{T} U\left(X_{N}\right) d B_{t}+\phi(t) d t
$$

where $\phi(t) \leq 0$ is an adapted process.

Proof. Define the Lyapunov function

$$
V(X)=\left(x_{1}^{2}+x_{2}^{2}+x_{3}^{2}\right)^{p / 2}=\|X\|_{2}^{p} .
$$

Using the Itŏ formula with respect to $V\left(X_{N}\right)$ for $p \in \mathbb{N}$ is even, we compute the Itŏ derivative of the Lyapunov function of the solution of the modified system. We consider

$$
\begin{aligned}
& d U\left(X_{N}\right)=\sum_{i=1}^{3} \frac{p}{2}\left(x_{N 1}^{2}+x_{N 2}^{2}+x_{N 3}^{2}\right)^{p / 2-1} 2 x_{N i} d x_{N i} \\
& +\frac{1}{2} \sum_{i, j=1}^{3} \frac{p}{2}\left(\frac{p}{2}-1\right)\left(x_{N 1}^{2}+x_{N 2}^{2}+x_{N 3}^{2}\right)^{p / 2-2} \\
& \times 2 x_{N i} 2 x_{N j} d x_{N i} d x_{N j} \\
& +\frac{1}{2} \sum_{i=1}^{3} \frac{p}{2}\left(x_{N 1}^{2}+x_{N 2}^{2}+x_{N 3}^{2}\right)^{p / 2-1} 2 d x_{N i} d x_{N i} \\
& =p\left\|X_{N}\right\|_{2}^{p-2}\left(d X_{N}, X_{N}\right) \\
& +p\left(\frac{p}{2}-1\right)\left\|X_{N}\right\|_{2}^{p-4} \sum_{i, j=1}^{3} x_{N i} d x_{N i} x_{N j} d x_{N j} \\
& +\frac{p}{2}\left\|X_{N}\right\|_{2}^{p-2} \sum_{i=1}^{3} d x_{N i} d x_{N i} \\
& =p\left\|X_{N}\right\|_{2}^{p-2}\left\{-\left[\left(A X_{N}, X_{N}\right)\right.\right. \\
& +\left(\chi_{N}\left(X_{N}\right) C\left(X_{N}\right), X_{N}\right) \\
& \left.\left.-\left(F, X_{N}\right)\right]\right\} d t \\
& +p\left(\frac{p}{2}-1\right) \\
& \times\left\|X_{N}\right\|_{2}^{p-4} \operatorname{trace}\left(X_{N} X_{N}^{T} U\left(X_{N}\right) U^{T}\left(X_{N}\right)\right) d t \\
& +\frac{p}{2}\left\|X_{N}\right\|_{2}^{p-2} \operatorname{trace}\left(U\left(X_{N}\right) U^{T}\left(X_{N}\right)\right) d t \\
& +p\left\|X_{N}\right\|_{2}^{p-2} X_{N}^{T} U\left(X_{N}\right) d B_{t} .
\end{aligned}
$$

We individually deal with all the right terms of (12). Firstly, from the definition of trace and Hölder inequality, we have the following.

Lemma 6. For any real matrix $R \in \mathbb{R}^{m \times n}, Q \in \mathbb{R}^{n \times m}$, the following inequalities hold:

$$
\|R Q\|_{2} \leq\|R\|_{2}\|Q\|_{2}, \quad|\operatorname{trace}(R Q)| \leq\|R\|_{2}\|Q\|_{2} .
$$


Proof. From Hölder inequality, it follows that

$$
\begin{gathered}
\|R Q\|_{2}^{2}=\sum_{i j}\left(\sum_{k} r_{i k} q_{k j}\right)^{2} \leq \sum_{i, j}\left(\sum_{k} r_{i k}^{2} \sum_{l} q_{l j}^{2}\right) \\
=\sum_{i, j, k, l}\left(r_{i k}^{2} q_{l j}^{2}\right)=\sum_{i, k} r_{i k}^{2} \sum_{j, l} q_{l j}^{2}=\|R\|_{2}^{2}\|Q\|_{2}^{2}, \\
|\operatorname{trace}(R Q)|^{2}=\left(\sum_{i, j} r_{i j} q_{j i}\right)^{2} \leq \sum_{i, j} r_{i j}^{2} \sum_{i, j} q_{j i}^{2}=\|R\|_{2}^{2}\|Q\|_{2}^{2} .
\end{gathered}
$$

By Lemma 6, trace $\left(X_{N} X_{N}^{T} U\left(X_{N}\right) U^{T}\left(X_{N}\right)\right)$ of (12) can be estimated,

$$
\begin{aligned}
\operatorname{trace} & \left(X_{N} X_{N}^{T} U\left(X_{N}\right) U^{T}\left(X_{N}\right)\right) \\
& \leq\left\|X_{N} X_{N}^{T}\right\|_{2}\left\|U\left(X_{N}\right) U^{T}\left(X_{N}\right)\right\|_{2} \\
& \leq\left\|X_{N}\right\|_{2}^{2}\|U(X)\|_{2}^{2} .
\end{aligned}
$$

Combining (A2) and Hölder inequality, we have

$$
-\left(A X_{N}, X_{N}\right)+\left(F, X_{N}\right) \leq-\frac{\lambda}{2}\left\|X_{N}\right\|_{2}^{2}+\frac{b^{2}}{2 \lambda} .
$$

From (A3), we can compute

$$
\begin{gathered}
\left(\chi_{N}\left(X_{N}\right) C\left(X_{N}\right), X_{N}\right) \\
=\left(\chi_{N}\left(X_{N}\right)\left(\begin{array}{c}
-\alpha_{3}(t) x_{N 3} x_{N 2}+\alpha_{3}(t) x_{N 3}^{2} \\
\alpha_{1}(t) x_{N 1} x_{N 3} \\
-\alpha_{2}(t) x_{N 1} x_{N 2}-\alpha_{3}(t) x_{N 1} x_{N 3}
\end{array}\right),\left(\begin{array}{l}
x_{N 1} \\
x_{N 2} \\
x_{N 3}
\end{array}\right)\right) \\
=\chi_{N}\left(X_{N}\right)\left(-\alpha_{3}(t) x_{N 3} x_{N 2} x_{N 1}+\alpha_{3}(t) x_{N 3}^{2} x_{N 1}\right. \\
+\alpha_{1}(t) x_{N 1} x_{N 3} x_{N 2}-\alpha_{2}(t) x_{N 1} x_{N 2} x_{N 3} \\
\left.-\alpha_{3}(t) x_{N 1} x_{N 3}^{2}\right) \equiv 0 .
\end{gathered}
$$

Using Lemma 6 again and combining all the above estimations from (15)-(17), we derive that

$$
\begin{aligned}
d\left\|X_{N}\right\|_{2}^{p}= & -\frac{p \lambda}{2}\left\|X_{N}\right\|_{2}^{p} d t+p\left\|X_{N}\right\|_{2}^{p-2} \frac{b^{2}}{2 \lambda} d t \\
& +0+p\left(\frac{p}{2}-1\right)\left\|X_{N}\right\|_{2}^{p-2}\left\|U\left(X_{N}\right)\right\|_{2}^{2} d t \\
& +\frac{p}{2}\left\|X_{N}\right\|_{2}^{p-2}\left\|U\left(X_{N}\right)\right\|_{2}^{2} d t \\
& +p\left\|X_{N}\right\|_{2}^{p-2} X_{N}^{T} U\left(X_{N}\right) d B_{t}+\phi(t) d t,
\end{aligned}
$$

where $\phi(t) \leq 0$ is an adapted process, which compensates all the estimations we made. The proof of Lemma 5 is completed.

Lemma 7. Assume that the conditions (A1)-(A3) hold and let $p \in \mathbb{N}$ be even and fixed, the initial expectation $E\left\|X_{0}\right\|_{2}^{p}<\infty$. Then

$$
E\left\|X_{N}(t)\right\|_{2}^{p} \leq C_{p}, \quad \forall t \in[0, T]
$$

where $C_{p}$ is a constant and only dependent on $T, E\left\|X_{0}\right\|_{2}^{p}, p$, $\lambda, C_{1}$, and $b$, but independent of $N$.

Proof. Firstly, we introduce the stopping time. For $D \in \mathbb{N}$, let

$$
\tau_{D}:=\inf \left\{t \in[0, T]:\left\|X_{N}(t)\right\|_{2} \geq D\right\} .
$$

Note that, for all $f(\cdot) \geq 0$,

$$
\int_{0}^{t \wedge \tau_{D}} f(s) d s \leq \int_{0}^{t} f\left(s \wedge \tau_{D}\right) d s .
$$

For any $t \in[0, T]$, using the linear growth condition of $U(X)$, we can integrate (10) from 0 to $t \wedge \tau_{D}$ and then take the expectations to obtain

$$
\begin{aligned}
E\left\|X_{N}\left(t \wedge \tau_{D}\right)\right\|_{2}^{p} \\
\leq E\|X(0)\|_{2}^{p}+E \int_{0}^{t \wedge \tau_{D}}\left(-\frac{p \lambda}{2}\right)\left\|X_{N}(s)\right\|_{2}^{p} d s \\
+E \int_{0}^{t \wedge \tau_{D}} \frac{p b^{2}}{2 \lambda}\left\|X_{N}(s)\right\|_{2}^{p-2} d s \\
+\frac{p(p-1)}{2} E \int_{0}^{t \wedge \tau_{D}} C_{1}\left\|X_{N}(s)\right\|_{2}^{p-2} d s \\
+\frac{p(p-1)}{2} C_{1} E \int_{0}^{t \wedge \tau_{D}}\left\|X_{N}(s)\right\|_{2}^{p} d s+0 \\
=E\|X(0)\|_{2}^{p} \\
+E \int_{0}^{t \wedge \tau_{D}}\left(\frac{C_{1} p(p-1)}{2}-\frac{p \lambda}{2}\right)\left\|X_{N}(s)\right\|_{2}^{p} d s \\
\quad+E \int_{0}^{t \wedge \tau_{D}}\left(\frac{C_{1} p(p-1)}{2}+\frac{p b^{2}}{2 \lambda}\right)\left\|X_{N}(s)\right\|_{2}^{p-2} d s \\
\quad+\int_{0}^{t}\left(\frac{C_{1} p(p-1)}{2}+\frac{p b^{2}}{2 \lambda}\right) E\left\|X_{N}\left(s \wedge \tau_{D}\right)\right\|_{2}^{p-2} d s . \\
\quad E\|X(0)\|_{2}^{p}+\int_{0}^{t}\left|\frac{C_{1} p(p-1)}{2}-\frac{p \lambda}{2}\right| E X_{N}(s) \|_{2}^{p} d s
\end{aligned}
$$

Let $p=2$ and use the Gronwall inequalities, then there exists a constant $C_{2}$, such that

$$
\sup _{t \in[0, T]} E\left\|X_{N}\left(t \wedge \tau_{D}\right)\right\|_{2}^{p} \leq C_{2} .
$$

Computing recursively, we obtain that there exists a constant $C_{p}$, such that

$$
\begin{aligned}
E\left\|X_{N}\left(t \wedge \tau_{D}\right)\right\|_{2}^{p} & \\
\leq E\left\|X_{0}\right\|_{2}^{p}+\int_{0}^{t} & \left(\left|\frac{C_{1} \mathrm{p}(p-1)}{2}-\frac{p \lambda}{2}\right| E\left\|X_{N}\left(s \wedge \tau_{D}\right)\right\|_{2}^{p}\right. \\
& \left.+\left(\frac{C_{1} p(p-1)}{2}+\frac{p b^{2}}{2 \lambda}\right) C_{p-2}\right) d s \leq C_{p} .
\end{aligned}
$$




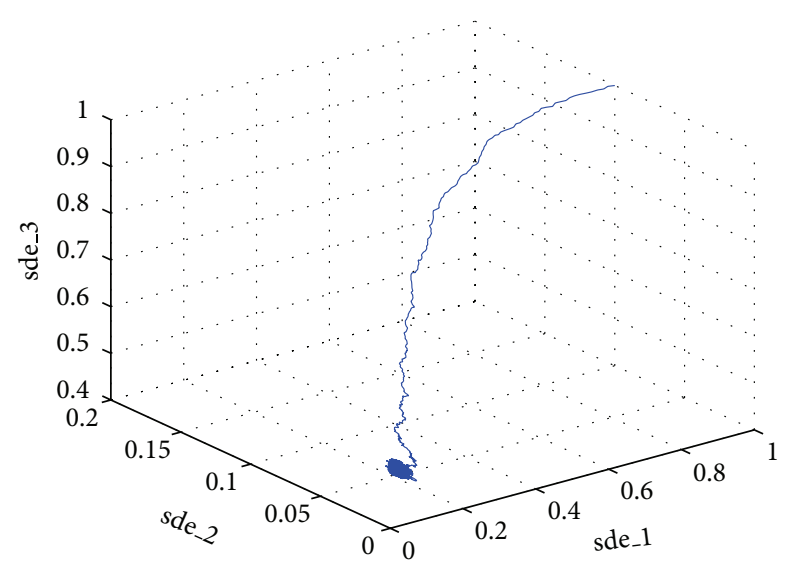

(a)
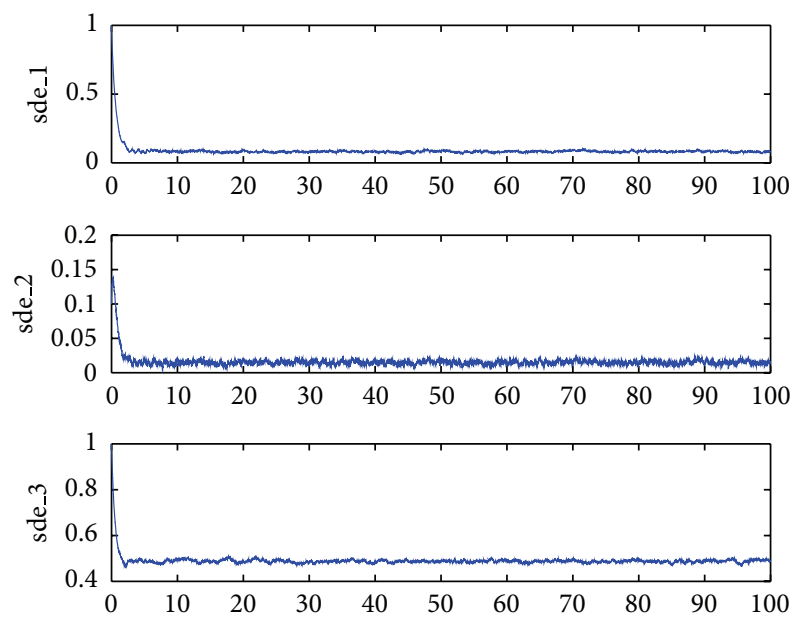

(b)

FIgURE 7: The attractor and the time series of the Stochastic LorentzRössler combining system with a white noise $u_{i i}=0.01, i=1,2,3$.

It is obvious that the stopping time satisfies $\tau_{D} \rightarrow T$ as $D \rightarrow$ $\infty$. By the continuity of the solution $X_{N}(t)$ in $t$, we derive that $\left\|X_{N}\left(t \wedge \tau_{D}\right)\right\|_{2}^{p}$ is bounded. Therefore,

$$
\left\|X_{N}\left(t \wedge \tau_{D}\right)\right\|_{2}^{p} \longrightarrow\left\|X_{N}(t)\right\|_{2}^{p}, \quad \text { a.s. }(D \longrightarrow \infty)
$$

Combining (24), (25) and Fuatou lemma, we have

$$
\begin{aligned}
E\left\|X_{N}(t)\right\|_{2}^{p} & =E \lim _{D \rightarrow \infty}\left\|X_{N}\left(t \wedge \tau_{D}\right)\right\|_{2}^{p} \\
& \leq \lim _{D \rightarrow \infty} \inf E\left\|X_{N}\left(t \wedge \tau_{D}\right)\right\|_{2}^{p} \leq C_{p} .
\end{aligned}
$$

Lemma 8. Let $p \in \mathbb{N}$ be even, $E\left\|X_{0}\right\|_{2}^{p}<\infty$, and (A1)-(A3) hold. Then there exists a constant $\widetilde{C}_{p}$ such that

$$
E \sup _{t \in[0, T]}\left\|X_{N}(t)\right\|_{2}^{p} \leq \widetilde{C}_{p}, \quad \forall t \in[0, T]
$$

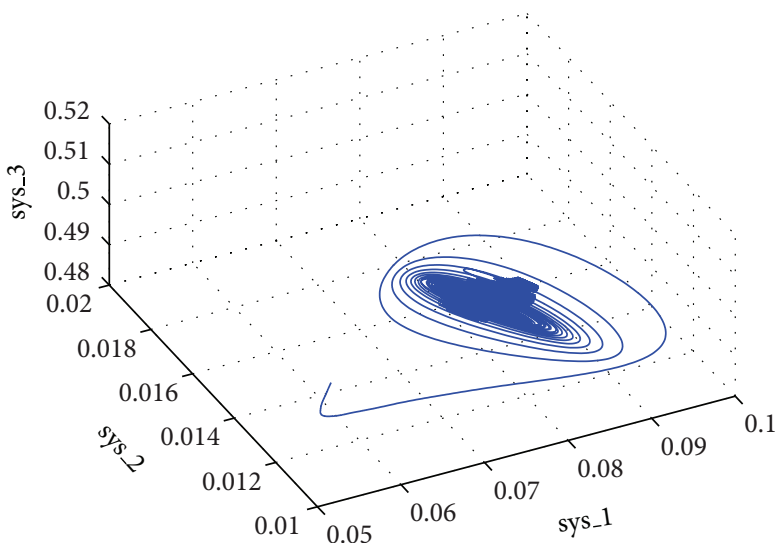

(a)
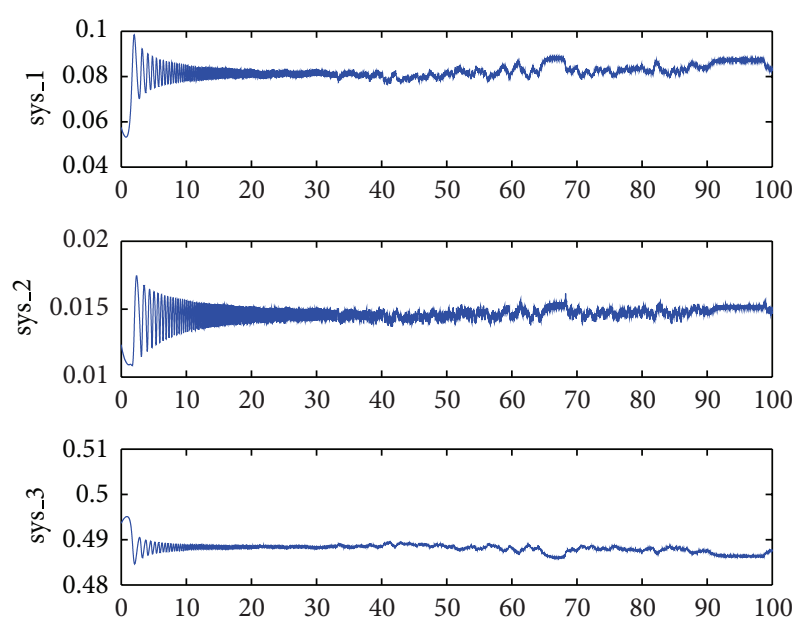

(b)

FIgURE 8: The attractor and the time series of the deterministic Lorentz-Rössler system.

where $\widetilde{C}_{p}$ is independent of $N$, only dependent on $T, E\left\|x_{0}\right\|_{2}^{p}$, $p, C_{1}, \lambda$, and $b$.

Proof. Note that $\tau_{D}$ is the stopping time introduced in (20). Integrating (10) (we take $p=2$ ),

$$
\begin{aligned}
\left\|X_{N}\left(t \wedge \tau_{D}\right)\right\|_{2}^{2}= & \left\|X_{0}\right\|_{2}^{2}+\int_{0}^{t \wedge \tau_{D}}\left(-\lambda\left\|X_{N}(s)\right\|_{2}^{2}\right) d s \\
& +\int_{0}^{t \wedge \tau_{D}} \frac{b^{2}}{\lambda} d s+\int_{0}^{t \wedge \tau_{D}}\left\|U\left(X_{N}(s)\right)\right\|_{2}^{2} d s \\
& +\int_{0}^{t \wedge \tau_{D}} 2 X_{N}^{T}(s) U\left(X_{N}(s)\right) d B_{s} \\
& +\int_{0}^{t \wedge \tau_{D}} \phi(s) d s .
\end{aligned}
$$

To estimate the expected supremum of $\left\|X_{N}\left(t \wedge \tau_{D}\right)\right\|_{2}^{p}$, we omit the nonpositive terms $\int_{0}^{t \wedge \tau_{D}}\left(-\lambda\left\|X_{N}(s)\right\|_{2}^{2}\right) d s$ and $\int_{0}^{t \wedge \tau_{D}} \phi(s) d s$, 


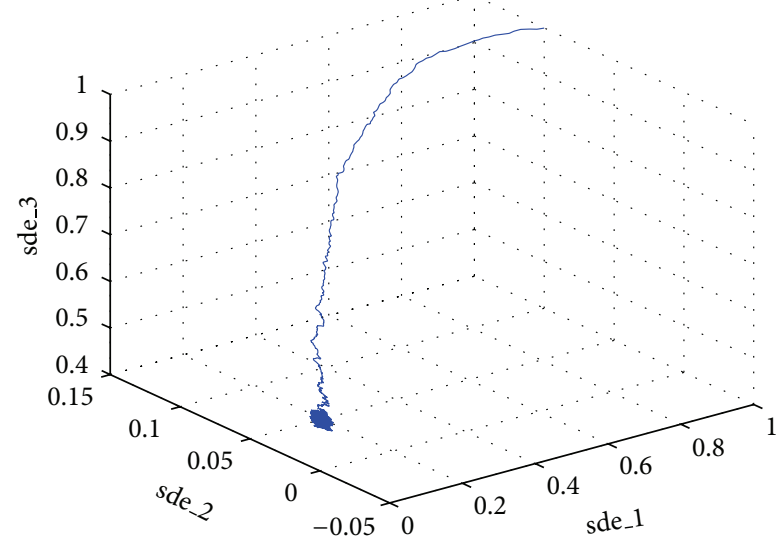

(a)
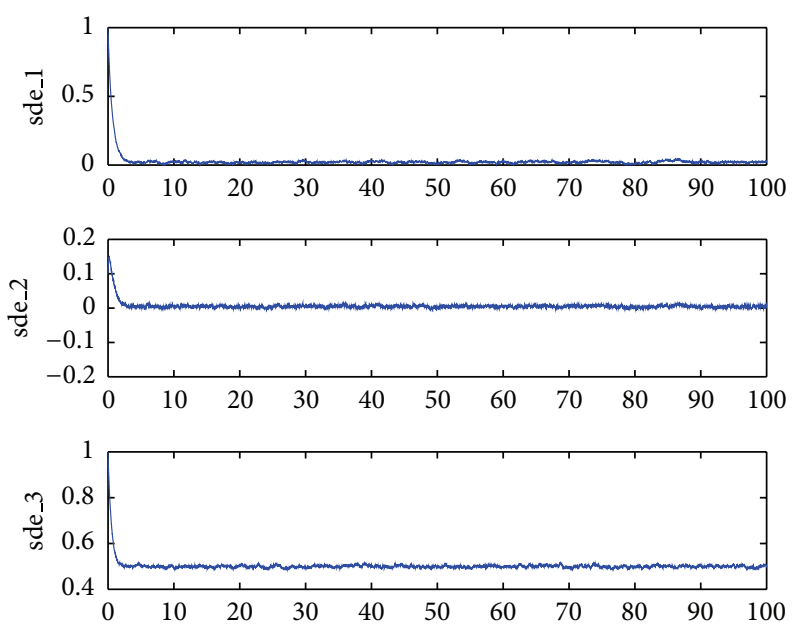

(b)

FIGURE 9: The attractor and the time series of the Stochastic LorentzRössler combining system with a white noise $u_{i i}=0.01, i=1,2,3$.

then

$$
\begin{aligned}
& \left\|X_{N}\left(t \wedge \tau_{D}\right)\right\|_{2}^{2} \\
& \leq\left\|X_{0}\right\|_{2}^{2}+\int_{0}^{t \wedge \tau_{D}} \frac{b^{2}}{\lambda} d s \\
& \quad+\int_{0}^{t \wedge \tau_{D}}\left\|U\left(X_{N}(s)\right)\right\|_{2}^{2} d s \\
& \quad+\int_{0}^{t \wedge \tau_{D}} 2 X_{N}^{T}(s) U\left(X_{N}(s)\right) d B_{s} .
\end{aligned}
$$

To obtain the estimation of $\left\|X_{N}\left(t \wedge \tau_{D}\right)\right\|_{2}^{p}$, we take the $p / 2$ power to (29) and use the primary inequality $\left|\sum_{i=1}^{n} a_{i}\right|^{k} \leq n^{k-1} \sum_{i=1}^{n}\left|a_{i}\right|^{k}$ for $k \geq 1$ (especially, let $n=3$,

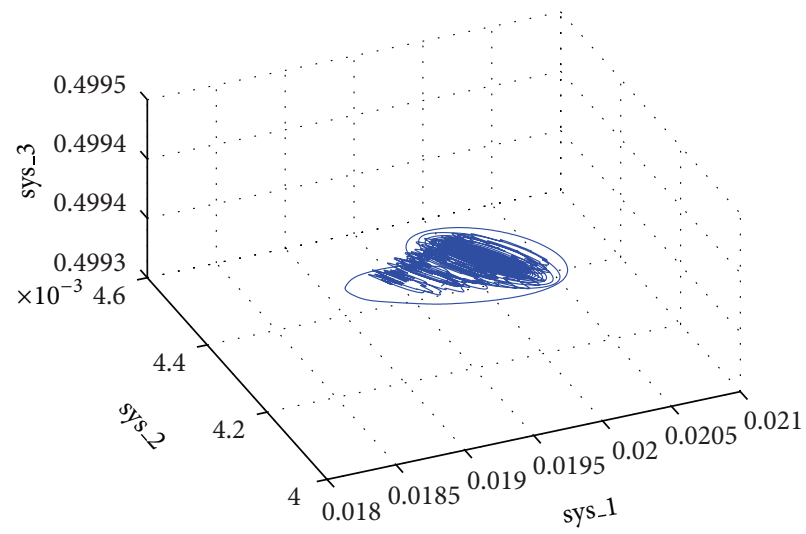

(a)
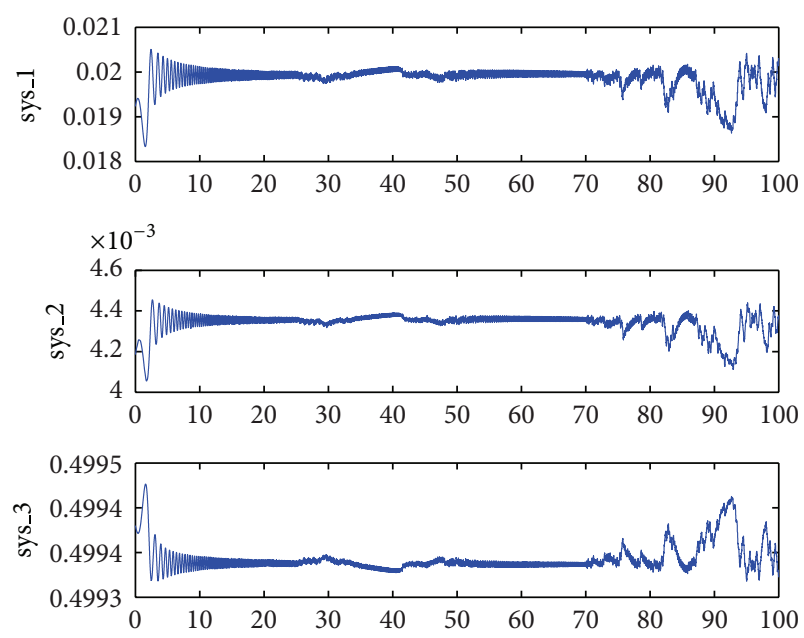

(b)

FIGURE 10: The attractor and the time series of the deterministic Lorentz-Rössler system.

$k=p / 2)$

$$
\begin{aligned}
\left\|X_{N}\left(t \wedge \tau_{D}\right)\right\|_{2}^{p} \leq & 3^{(p / 2-1)}\left\|X_{0}\right\|_{2}^{p} \\
& +3^{(p / 2-1)} \\
& \times\left|\int_{0}^{t \wedge \tau_{D}}\left(\frac{b^{2}}{\lambda}+\left\|U\left(X_{N}(s)\right)\right\|_{2}^{2}\right) d s\right|^{p / 2} \\
& +3^{(p / 2-1)} \\
& \times\left|\int_{0}^{t \wedge \tau_{D}} 2 X_{N}^{T}(s) U\left(X_{N}(s)\right) d B_{s}\right|^{p / 2}
\end{aligned}
$$

We now compute the expected supremum

$$
\begin{gathered}
E \sup _{t \in[0, T]}\left\|X_{N}\left(t \wedge \tau_{D}\right)\right\|_{2}^{p} \\
\leq 3^{(p / 2-1)} E\left\|X_{0}\right\|_{2}^{p}
\end{gathered}
$$




$$
\begin{aligned}
& +3^{(p / 2-1)} E \\
& \times\left|\int_{0}^{T}\left(\frac{b^{2}}{\lambda}+\left\|U\left(X_{N}(s)\right)\right\|_{2}^{2}\right) d s\right|^{p / 2} \\
& +3^{(p / 2-1)} E \\
& \times \sup _{t \in[0, T]}\left|\int_{0}^{t \wedge \tau_{D}} 2 X_{N}^{T}(s) U\left(X_{N}(s)\right) d B_{s}\right|^{p / 2} .
\end{aligned}
$$

To deal with the term $E\left|\int_{0}^{T}\left(b^{2} / \lambda+\left\|U\left(X_{N}(s)\right)\right\|_{2}^{2}\right) d s\right|^{p / 2}$, we use Hölder inequality, the linear growth condition (A1), and the primary inequality again,

$$
\begin{aligned}
\left.E||_{0}^{T}\left(\frac{b^{2}}{\lambda}+\left\|U\left(X_{N}(s)\right)\right\|_{2}^{2}\right) d s\right|^{p / 2} & \mid\left(\int_{0}^{T}\left(\frac{b^{2}}{\lambda}+C_{1}+C_{1}\left\|X_{N}(s)\right\|_{2}^{2}\right)^{p / 2} d s\right)^{2 / p} \\
\leq & \times\left.\left(\int_{0}^{T} 1^{p /(p-2)} d s\right)^{(p-2) / p}\right|^{p / 2} \\
\leq & T^{(p-2) / 2} E \int_{0}^{T}\left(\frac{b^{2}}{\lambda}+C_{1}+C_{1}\left\|X_{N}(s)\right\|_{2}^{2}\right)^{p / 2} d s \\
\leq & 3^{p / 2-1} T^{(p-2) / 2} \\
& \times E \int_{0}^{T}\left(\left(\frac{b^{2}}{\lambda}\right)^{p / 2}+C_{1}^{p / 2}+C_{1}^{p / 2}\left\|X_{N}(s)\right\|_{2}^{p}\right) d s .
\end{aligned}
$$

Note that the solution of the modified system is both continuous in both $t$ and $\mathscr{F}_{t}$ measurable for all $t \geq 0$. By Fubini theorem and the boundary of Lemma 7, we have

$$
\begin{aligned}
& E\left|\int_{0}^{T}\left(\frac{b^{2}}{\lambda}+\left\|U\left(X_{N}(s)\right)\right\|_{2}^{2}\right) d s\right|^{p / 2} \\
& \quad \leq(3 T)^{(p-2) / 2} \int_{0}^{T}\left(\left(\frac{b^{2}}{\lambda}\right)^{p / 2}+C_{1}^{p / 2}+C_{1}^{p / 2} C_{p}\right) d s:=\widetilde{C}_{p}^{(1)} .
\end{aligned}
$$

We now use the Burholder-Davis-Gundy inequality ([18, Theorem 2.6]) to estimate the stochastic integral

$$
\begin{aligned}
E \sup _{t \in[0, T]}\left|\int_{0}^{t \wedge \tau_{D}} 2 X_{N}^{T}(s) U\left(X_{N}(s)\right) d B_{s}\right|^{p / 2} \\
\quad \leq \widetilde{C}_{p}^{(2)} E\left|\int_{0}^{T}\left\|X_{N}^{T}(s) U\left(X_{N}(s)\right)\right\|_{2}^{2} d s\right|^{p / 4},
\end{aligned}
$$

with

$$
\widetilde{C}_{p}^{2}:= \begin{cases}\left(\frac{64}{p}\right)^{p / 4}, & 0<p<4 \\ \frac{(p / 2)^{p / 2+1}}{2(p / 2-1)^{p / 2-1}}, & 4 \geq p .\end{cases}
$$

If $p=2$, we use the inequality $\sqrt{x} \leq 1+x$. For $p>2$, we use the Hölder inequality to remove the power outside the integral. Afterwards we proceed a similar technique as we handled the term $\int_{0}^{T}\left\|U\left(X_{N}(s)\right)\right\|_{2}^{2} d s$, which leads to a constant $\widetilde{C}_{p}^{(3)}$, such that

$$
E \sup _{t \in[0, T]}\left|\int_{0}^{t \wedge \tau_{D}} 2 X_{N}^{T}(s) U\left(X_{N}(s)\right) d B_{s}\right|^{p / 2} \leq \widetilde{C}_{p}^{(3)} .
$$

Combine (31)-(36), then it follows that

$$
\begin{aligned}
& E \sup _{t \in[0, T]}\left\|X_{N}\left(t \wedge \tau_{D}\right)\right\|_{2}^{p} \\
& \quad \leq 3^{(p-2) / 2} E\left\|X_{0}\right\|_{2}^{p}+3^{(p-2) / 2} \widetilde{C}_{p}^{(1)}+3^{(p-2) / 2} \widetilde{C}_{p}^{(3)}:=\widetilde{C}_{p} .
\end{aligned}
$$

To complete the proof, we mention that $t \wedge \tau_{D} \rightarrow t$ for $D \rightarrow \infty$. Furthermore, we note that the solution $X_{N}(t)$ is continuous in $t$. Thus

$$
\sup _{t \in[0, T]}\left\|X_{N}\left(t \wedge \tau_{D}\right)\right\|_{2}^{p} \longrightarrow \sup _{t \in[0, T]}\left\|X_{N}(t)\right\|_{2}^{p}, \quad \text { a.s. }(D \longrightarrow \infty) \text {. }
$$

Since all terms are non negative and the limit exists, using Fatou lemma, we have

$$
\begin{aligned}
E \sup _{t \in[0, T]}\left\|X_{N}(t)\right\|_{2}^{p} & =E \lim _{D \rightarrow \infty} \sup _{t \in[0, T]}\left\|X_{N}\left(t \wedge \tau_{D}\right)\right\|_{2}^{p} \\
& \leq \lim \inf _{D \rightarrow \infty} E \sup _{t \in[0, T]}\left\|X_{N}\left(t \wedge \tau_{D}\right)\right\|_{2}^{p} \leq \widetilde{C}_{p} .
\end{aligned}
$$

The uniform boundary of the expected supremum is obtained.

In the following theorem, the main results are introduced by the estimations of the moments.

Theorem 9. Let (A1)-(A3) hold. Then the Lorentz-Rössler system given by (5) and (6) with $E\left\|X_{0}\right\|_{2}^{2}<\infty$ possesses a unique almost sure continuous solution process, which has the following properties:

If in addition $E\left\|X_{0}\right\|_{2}^{p}<\infty$ for a fixed $p \in \mathbb{N}$ is even, then there exist two constants $\widetilde{C}^{(1)}>0$ and $\widetilde{C}^{(2)}>0$, which are only dependent on $T, E\left\|X_{0}\right\|_{2}^{p}, p, \lambda$, and $b$, such that

$$
\begin{gathered}
E\|X(t)\|_{2}^{p} \leq \widetilde{C}^{(1)}, \quad \forall t \in[0, T], \\
E \sup _{t \in[0, T]}\|X(t)\|_{2}^{p} \leq \widetilde{C}^{(2)} .
\end{gathered}
$$




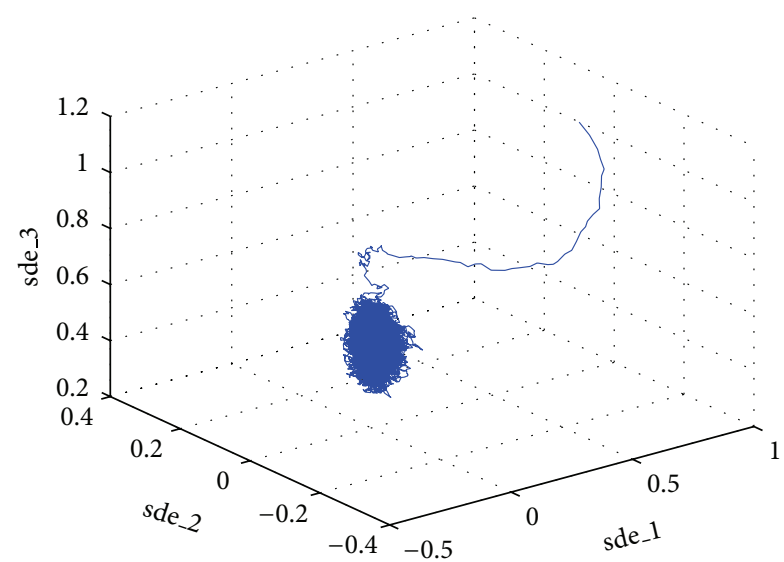

(a)
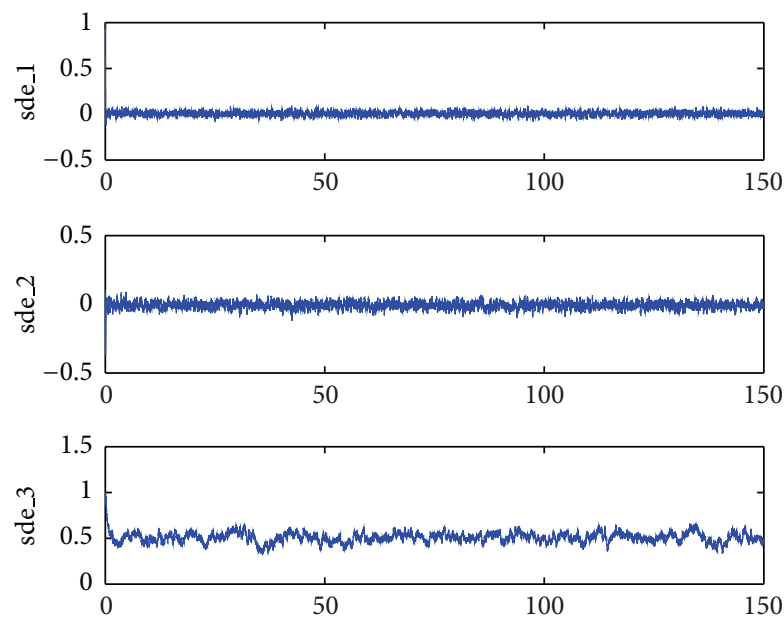

(b)

FIGURE 11: Stochastic system with a white noise $u_{i i}=0.1, i=1,2,3$.

Proof. Since the coefficients of the system (5) satisfy the local Lipschitz condition, the uniqueness follows. Furthermore, Lemma 3 exists a continuous solution $X_{N}(t)$. To prove the existence of Theorem 9, we need to show that $X_{N}(t) \rightarrow X(t)$ for $N \rightarrow \infty$.

Let $\tau_{D}$ denote the stopping time introduced in (20) for an $N \in \mathbb{N}$. Using Lemma 8 and Chebyshev inequality, we get

$$
\begin{aligned}
\mathbb{P}\left\{\tau_{N}(\omega)<T\right\} & \leq \mathbb{P}\left\{\sup _{t \in[0, T]}\left\|X_{N}(t)\right\|_{2} \geq N\right\} \\
& \leq \frac{E \sup _{t \in[0, T]}\left\|X_{N}(t)\right\|_{2}^{2}}{N^{2}} \\
& \leq \frac{\widetilde{C}\left(T, E\left\|X_{0}\right\|_{2}^{2}\right)}{N^{2}} \longrightarrow 0, \quad(N \longrightarrow \infty) .
\end{aligned}
$$

We can find for almost every $\omega \in \Omega$ and an $N_{0}(\omega)$ such that $\tau_{N_{0}(\omega)}=T$. Moreover, we have

$$
C_{N^{\prime}}(X)=C_{N}(X)=C(X), \quad N^{\prime} \geq N>0, \forall\|X\|_{2} \leq N .
$$

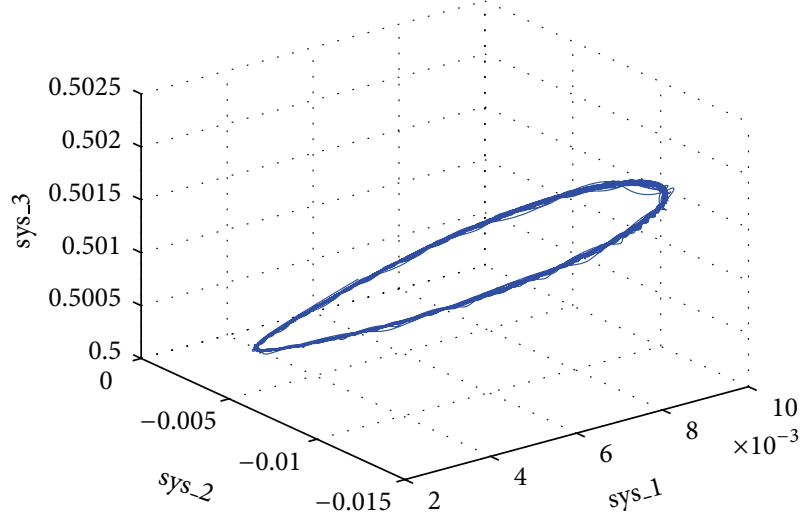

(a)
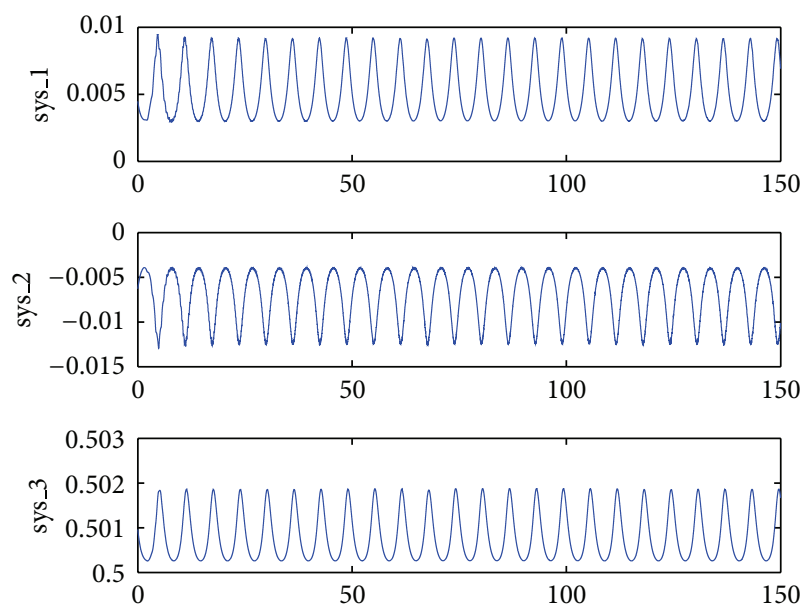

(b)

FIgURE 12: Deterministic system.

Thus

$$
\begin{array}{r}
\tau_{N^{\prime}} \geq \tau_{N}, \quad X_{N^{\prime}}^{X_{0}}(\cdot, \omega)= \\
X_{N}^{X_{0}}(\cdot, \omega) \text { (almost sure) }, \\
\text { on }\left[0, \tau_{N}\right] \forall N^{\prime} \geq N .
\end{array}
$$

From (43), if $\tau_{N}=T$, then $\tau_{N^{\prime}}=T$ for all $N^{\prime} \geq N$. Thus the set $\left\{\omega: \tau_{N}(\omega)=T\right\}$ is monotonously increasing and converges to $\Omega$ as $N \rightarrow \infty$. Combining (41), we have

$$
\tau_{N} \stackrel{\mathbb{P}}{\longrightarrow} T, \quad(N \longrightarrow \infty)
$$

Moreover, because $X_{N}(t)$ is continuous in $t$ and converges to $X(t)$ uniformly in $t, X(t)$ is also continuous in $t$. (Actually, note that if $\tau_{N_{0}(\omega)}=T$, then we can express for almost all $\omega \in \Omega$ the limit function by $X(\cdot, \omega):=X_{N^{\prime}}(\cdot, \omega)$ for all $N^{\prime} \geq$ $N_{0}(\omega)$.)

In the following proof, we have to show that the limit function is the solution of the original Lorentz-Rössler system. When $t=0$, it is obvious that $X_{N}(0)=X(0)=X_{0}$ 


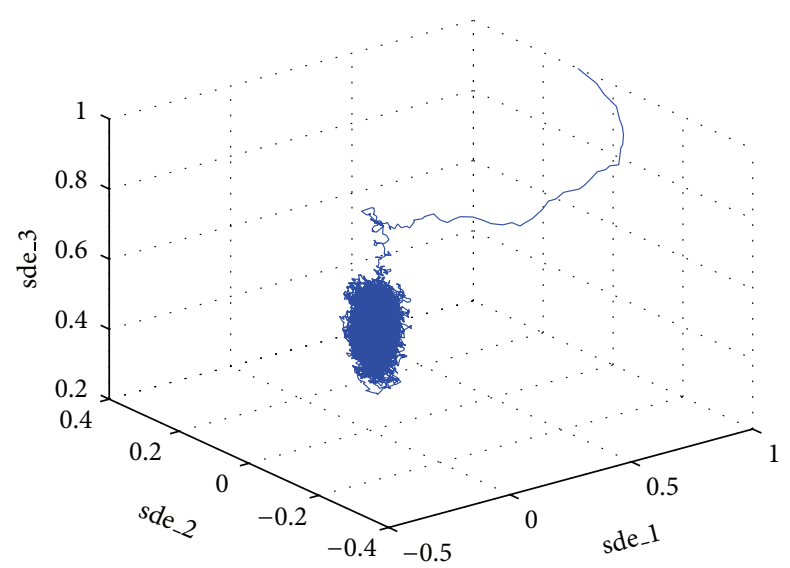

(a)
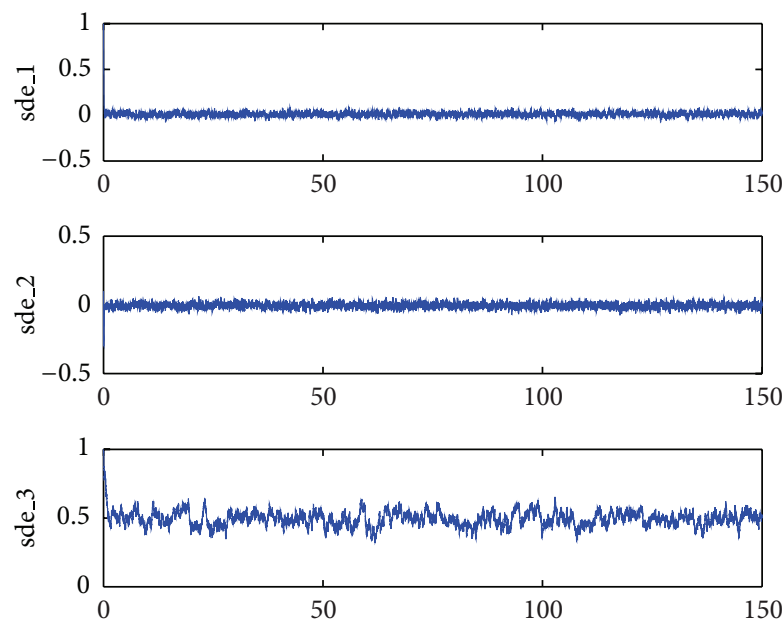

(b)

FIGURE 13: Transform linear terms system with a white noise $u_{i i}=$ $0.1, i=1,2,3$.

for all $N \in \mathbb{N}$. For $t \in(0, T]$, we consider the limit of (9) for $N \rightarrow \infty$. By the definition of $\tau_{D}$ in (20), it follows that

$$
\begin{aligned}
& C_{N}\left(X_{N}\left(t \wedge \tau_{N}\right)\right)=C\left(X\left(t \wedge \tau_{N}\right)\right), \\
& X_{N}\left(t \wedge \tau_{N}\right)=X\left(t \wedge \tau_{N}\right), \quad \forall t<T .
\end{aligned}
$$

Moreover the almost sure convergence of $\tau_{N} \stackrel{(N \rightarrow \infty)}{\longrightarrow} T$ implies

$$
\begin{gathered}
\mathbb{P}\left\{\sup _{t \in[0, T]} \| \int_{t_{0}}^{t}\left(A\left(X_{N}(s)-X(s)\right)+(F-F)\right.\right. \\
\left.\quad+C_{N}\left(X_{N}(s)\right)-C(X(s))\right) d s \\
\left.\quad+\int_{t_{0}}^{t}\left(U\left(X_{N}(s)\right)-U(X(s))\right) d B_{s} \|_{2}>0\right\} \\
\leq \mathbb{P}\left\{\tau_{N}<t\right\} \stackrel{(N \rightarrow \infty)}{\longrightarrow} 0 .
\end{gathered}
$$

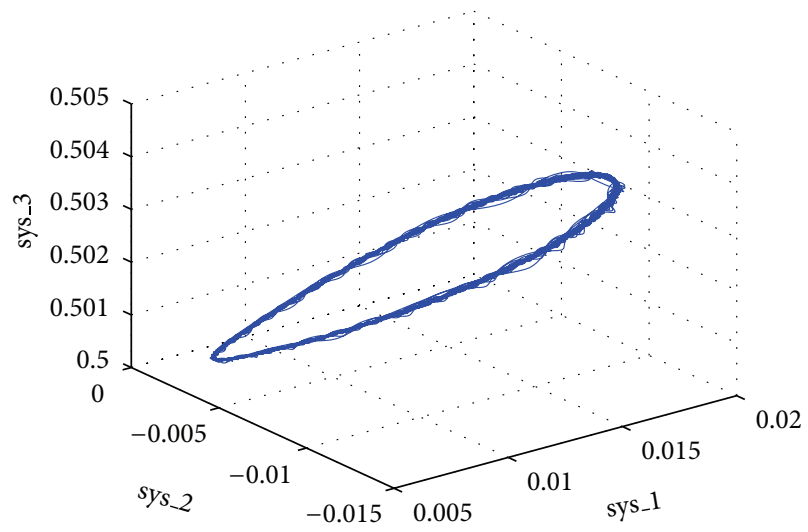

(a)
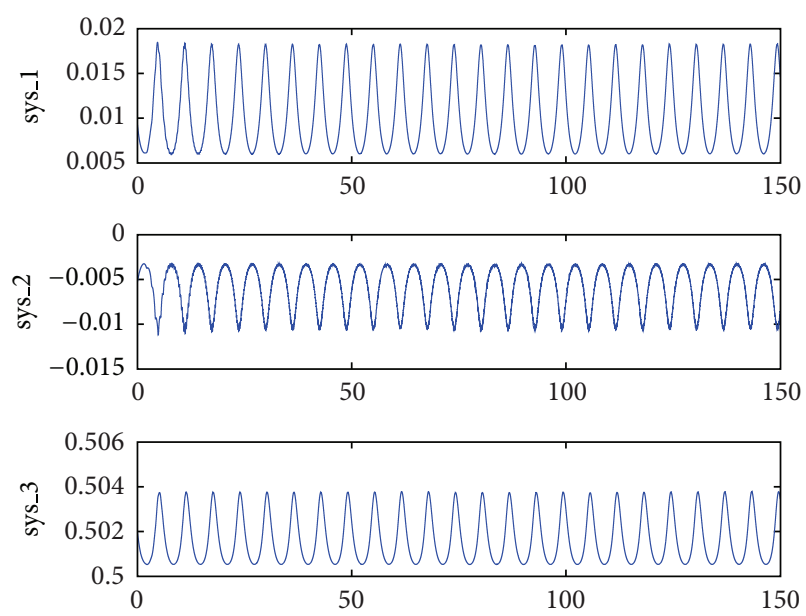

(b)

FIGURE 14: Deterministic system with different linear terms.

In fact, if $\tau_{N} \geq t$, then $\tau_{N} \wedge t=t$. So $X_{N}(t)=X(t)$, $C_{N}\left(X_{N}(t)\right)=C(X(t))$, and

$$
\begin{aligned}
\sup _{t \in[0, T]} \| \int_{t_{0}}^{t}\left(A\left(X_{N}(s)-X(s)\right)+(F-F)\right. & \\
& \left.+C_{N}\left(X_{N}(s)\right)-C(X(s))\right) d s \\
& +\int_{t_{0}}^{t}\left(U\left(X_{N}(s)\right)-U(X(s))\right) d B_{s} \|_{2}=0 .
\end{aligned}
$$

Hence $X(\cdot)$ is a solution of the stochastic Lorentz-Rössler system on $[0, T]$. Finally, the boundary of the moments (40) can be obtained by the uniform convergence of $X_{N}(t)$ to $X(t)$ in $t$, Lemmas 7 and 8.

\section{Numerical Results}

In this section, we give some numerical results with different parameters for which the stochastic and deterministic Lorentz-Rössler systems show qualitatively different behaviors and illustrate our theory. The respective numerical 


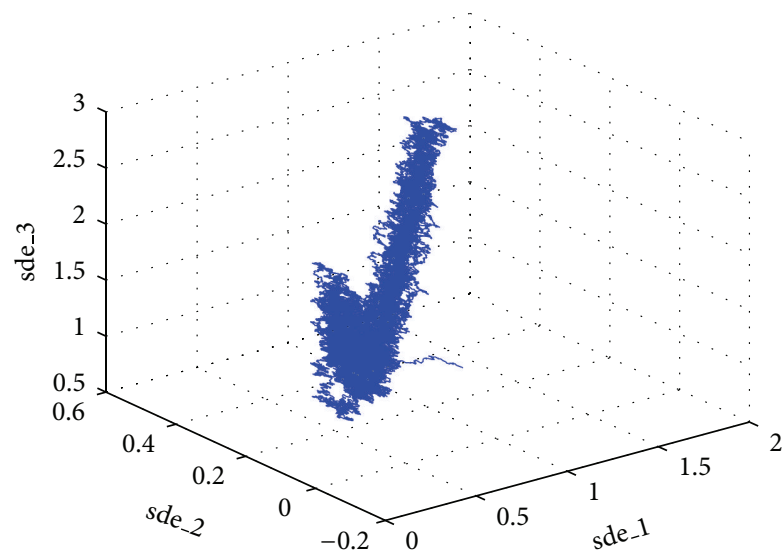

(a)
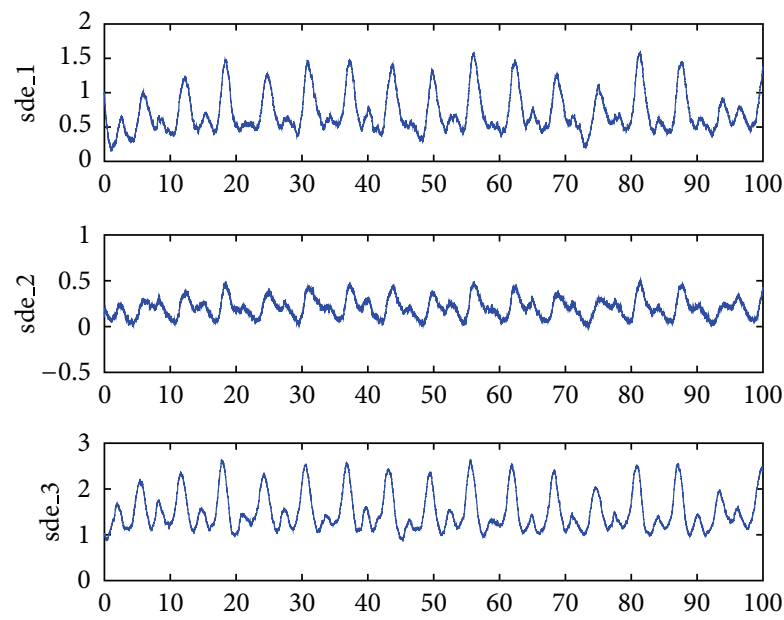

(b)

FIGURE 15: Stochastic system with a white noise $u_{i i}=0.1, i=1,2,3$.

schemes are the stochastic and deterministic Runge-Kutta numerical difference schemes. All of the initial value of our problems are given by $X_{0}=(1,0.1,1)$ and all of the systems of this section satisfy our conditions (A1)-(A3). To simplify, in this section, we only consider the independent noise $\left(u_{i j, i \neq j}=\right.$ $0, i, j=1,2,3)$.

Firstly, we consider some examples to exhibit the moment estimations of the Lorentz-Rössler combining systems.

Example 10. Let the coefficients of the linear terms be $\sigma=2$, $r=1, a=-7, b=1, \beta=2$, and $c=1$, and let nonlinear parameters be $\alpha_{1}(t)=2 /\left(\cos \left(t^{2}\right)+2\right), \alpha_{2}(t)=1 /\left(\cos \left(t^{2}\right)+2\right)$, $\alpha_{3}(\mathrm{t})=1 /\left(\cos \left(t^{2}\right)+2\right)$, which easily satisfy the conditions (A1)-(A3). The stochastic and deterministic (4) can be shown by Figures 7,8 .

Let the nonlinear terms be $\alpha_{1}(t)=4 /\left(\sin \left(t^{2}\right)+8\right), \alpha_{2}(t)=$ $3 /\left(\sin \left(t^{2}\right)+8\right)$, and $\alpha_{3}(t)=1 /\left(\sin \left(t^{2}\right)+8\right)$, then we can find that the stochastic and deterministic behavior make very different trajectory (Figures 9, 10).

Example 11. Let $c=0$. To satisfy the condition (A2), it is enough to take $\beta>0$ and $a<1$. Thus in this example, firstly,

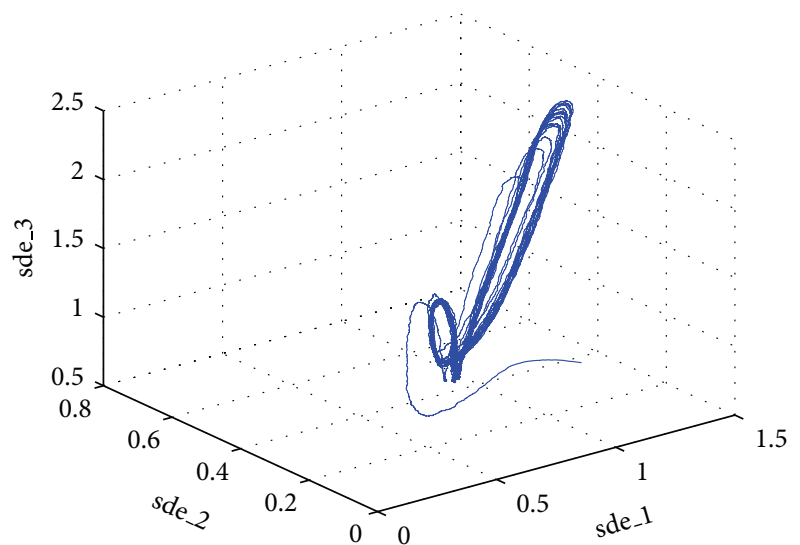

(a)
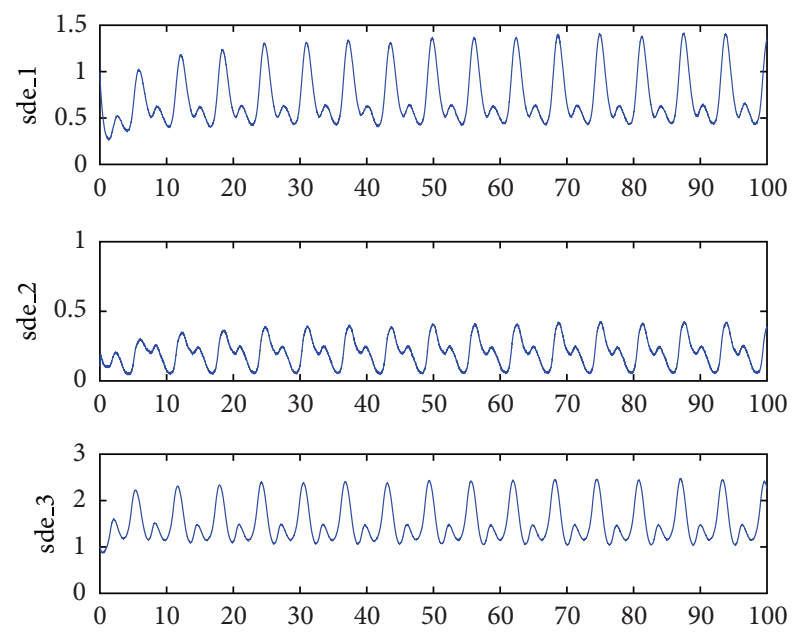

(b)

FIGURE 16: Stochastic system with a white noise $u_{i i}=0.01, i=1,2,3$.

we consider $\sigma=12, r=-10, a=-7, b=1, \beta=2$, and $c=0$ and $\alpha_{1}(t)=1 /(\sin t+2)+5 /\left(\sin \left(t^{2}\right)+2\right), \alpha_{2}(t)=5 /\left(\sin \left(t^{2}\right)+2\right)$, and $\alpha_{3}(t)=1 /(\sin t+2)$ (Figures 11, 12).

And in this example, we consider the effect of matrix $A$. Change the linear terms and take $\sigma=9, r=-10, a=-18$, $b=1, \beta=2$, and $c=0$ (Figures 13, 14).

Example 12. In this example, we mainly consider the effect of the noise. Let $\sigma=2, r=0, a=-18, b=1, \beta=|\cos t|$, $c=0$ and $\alpha_{1}(t)=t /(2 t+2)-((2 \sin t+3)+1 /(\cos (3 t)+2))$, $\alpha_{2}(t)=-((2 \sin t+3)+1 /(\cos (3 t)+2))$, and $\alpha_{3}(t)=t /(2 t+2)$ (Figures 15-18).

In this paper, we have established a sufficient condition under which the stochastic system (3) has a unique solution. And from these numerical results, some interesting qualities can be seen.

(i) From Examples 10-12, it is obvious that for any time $T$, Theorem 9 can be ensured. And the boundary of the moments of the solutions can be obtained.

(ii) Examples 10 and 11 show that for (4), the effect of the dynamical behavior would mainly depend on 


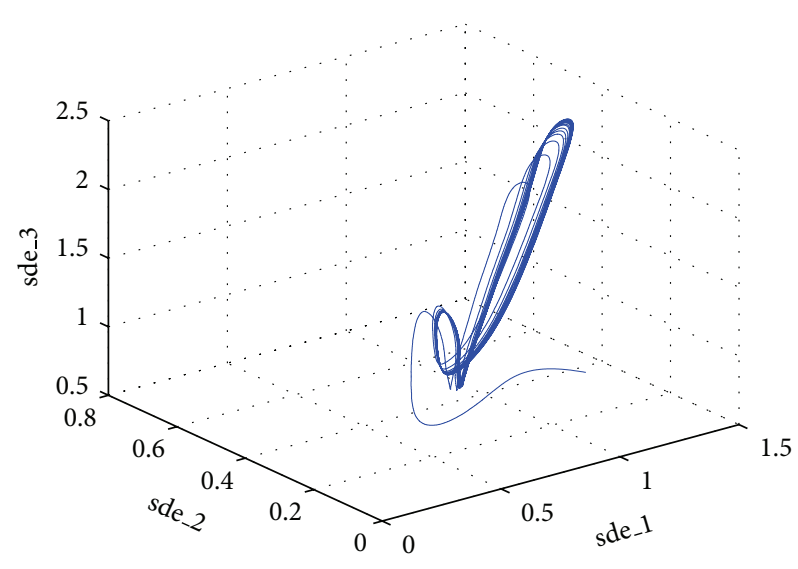

(a)
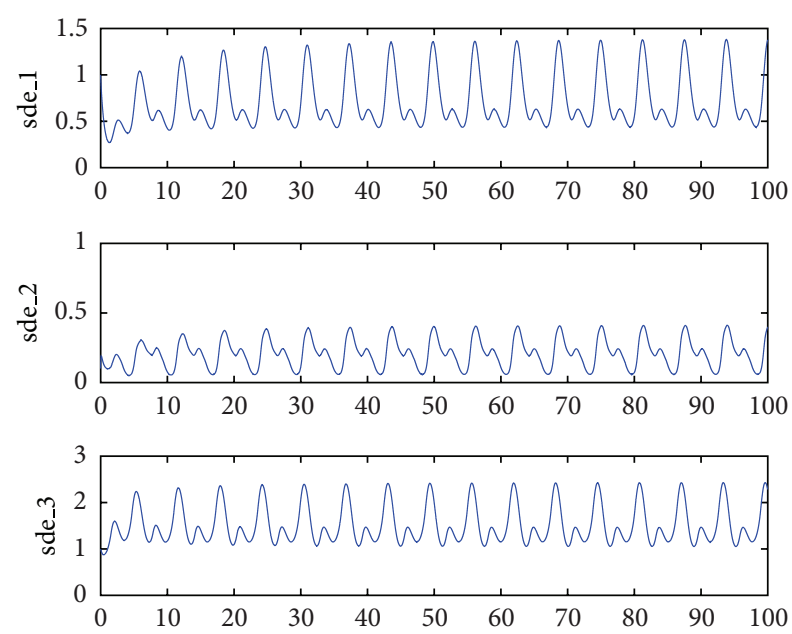

(b)

FIGURE 17: Stochastic system with a white noise $u_{i i}=0.001, i=$ $1,2,3$.

the nonlinear terms. Some transformations especially about linear terms are given by Example 11, but the paths have small transformations.

(iii) For the more general Lorentz-Rössler systems, if the uniqueness can be ensured, especially in Example 12, we can show that the stochastic systems converge toward the deterministic system when the intensity of noise converges to zero.

(iv) The system (3) gives us very abundant expressions including the behavior of the well-known Lorentz system and the Rössler system. Some systems with appropriate parameters in (3) can be used to make good hop-frequency time series.

\section{Acknowledgments}

This paper supported by NSFC (Grant no. 11101044), NSFC (Grant no. 11126257), and Special Funds for Co-construction Project of Beijing.

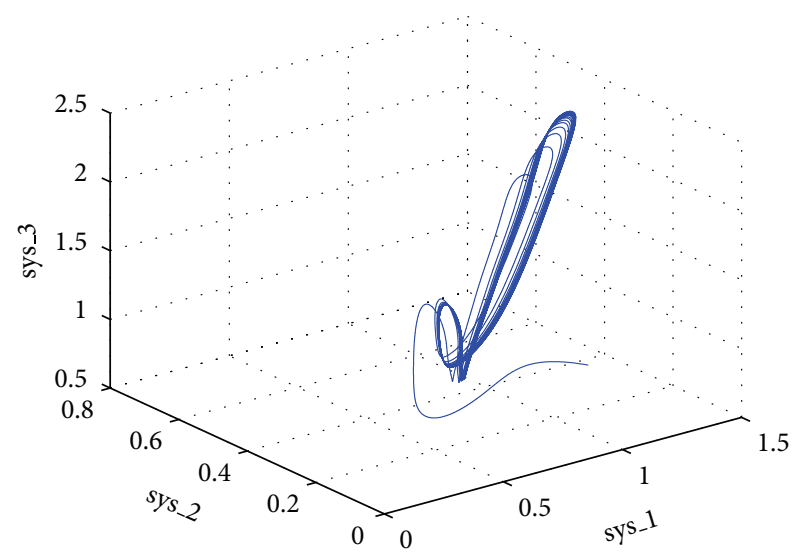

(a)
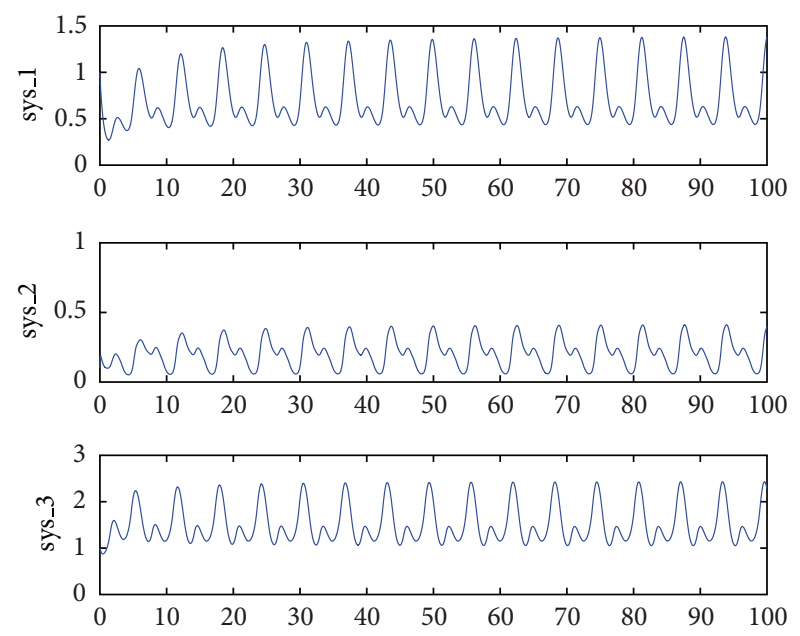

(b)

FIGURE 18: Deterministic system with a white noise $u_{i i}=0, i=$ $1,2,3$.

\section{References}

[1] E. N. Lorentz, "Determinstic nonperiodic flows," Journal of the Atmospheric Sciences, vol. 12, no. 20, pp. 130-141, 1963.

[2] H.-O. Peitgen, H. Jürgens, and D. Saupe, Chaos and Fractals, Springer, New York, NY, USA, 1992.

[3] H. Haken, "Analogy between higher instabilities in fluids and lasers," Physics Letters A, vol. 53, no. 1, pp. 77-78, 1975.

[4] M. Gorman, P. J. Widmann, and K. A. Robbins, "Nonlinear dynamics of a convection loop: a quantitative comparison of experiment with theory," Physica D, vol. 19, no. 2, pp. 255-267, 1986.

[5] N. Hemati, "Strange attractors in brushless DC motors," IEEE Transactions on Circuits and Systems I, vol. 41, no. 1, pp. 40-45, 1994.

[6] K. M. Cuomo, "Circuit implementation of synchronized chaos with application to communications," Physical Review Letters, vol. 71, no. 1, pp. 65-68, 1993.

[7] D. Poland, "Cooperative catalysis and chemical chaos: a chemical model for Lorentz equation," Physica D, vol. 65, no. 1, pp. 86-99, 1993.

[8] O. E. Rössler, "An equation for continuous chaos," Physics Letters A, vol. 57, no. 5, pp. 397-398, 1976. 
[9] M. Peifer, B. Schelter, M. Winterhalder, and J. Timmer, "Mixing properties of the Rãssler system and consequences for coherence and synchronization analysis," Physical Review E, vol. 72, no. 2, Article ID 026213, 7 pages, 2005.

[10] M. Itoh, T. Yang, and L. O. Chua, "Conditions for impulsive synchronization of chaotic and hyperchaotic systems," International Journal of Bifurcation and Chaos, vol. 11, no. 2, pp. 551560, 2001.

[11] S. H. Chen and J. Lü, "Synchronization of an uncertain unified chaotic systems using backstepping design," Chaos Solitions \& Fractals, vol. 14, pp. 643-647, 2002.

[12] J. Q. Fang, Y. Hong, and G. Chen, "Switching manifold approach to chaos synchronization," Physical Review E, vol. 59, no. 3, pp. 2523-2526, 1999.

[13] G. Chen and X. Dong, From Chaos to Order Methodologies Perspectives and Applications, World Scientific, Singapore, 1994.

[14] J. Zhu, J. Lü, and X. Yu, "Flocking of multi-agent non-holonomic systems with proximity graphs," IEEE Transactions on Circuits and Systems I, vol. 60, no. 1, pp. 199-210, 2013.

[15] P. Wang, J. Lü, and M. J. Ogorzalek, "Global relative parameter sensitivities of the feed-forward loops in genetic networks," Neurocomputing, vol. 78, no. 1, pp. 155-165, 2012.

[16] J. Lü and G. Chen, "A time-varying complex dynamical network model and its controlled synchronization criteria," IEEE Transactions on Automatic Control, vol. 50, no. 6, pp. 841-846, 2005.

[17] J. Lü, X. Yu, G. Chen, and D. Cheng, "Characterizing the synchronizability of small-world dynamical networks," IEEE Transactions on Circuits and Systems I, vol. 51, no. 4, pp. 787796, 2004.

[18] X. Mao, Exponential Stability of Stochastic Differential Equations, vol. 182 of Monographs and Textbooks in Pure and Applied Mathematics, Marcel Dekker, New York, NY, USA, 1994.

[19] M. Liu and K. Wang, "Persistence, extinction and global asymptotical stability of a non-autonomous predator-prey model with random perturbation," Applied Mathematical Modelling, vol. 36, no. 11, pp. 5344-5353, 2012.

[20] Q. Yang and D. Jiang, "A note on asymptotic behaviors of stochastic population model with Allee effect," Applied Mathematical Modelling, vol. 35, no. 9, pp. 4611-4619, 2011.

[21] S.-R. Cheng, "Stochastic population systems," Stochastic Analysis and Applications, vol. 27, no. 4, pp. 854-874, 2009.

[22] Z. Xing and J. Peng, "Boundedness, persistence and extinction of a stochastic non-autonomous logistic system with time delays," Applied Mathematical Modelling, vol. 36, no. 8, pp. 33793386, 2012. 


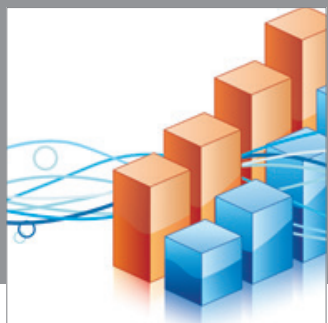

Advances in

Operations Research

mansans

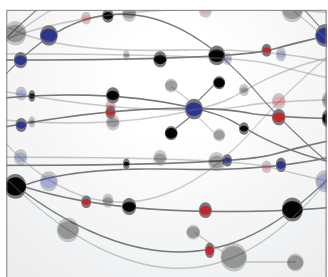

The Scientific World Journal
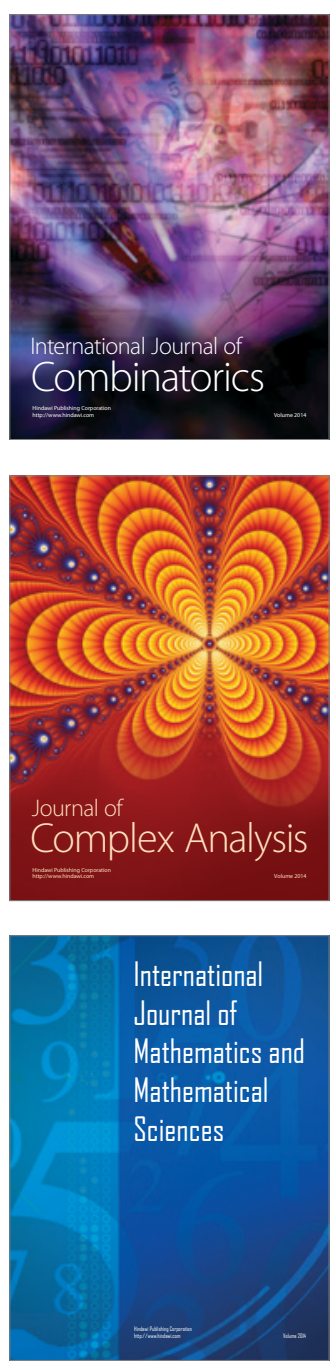
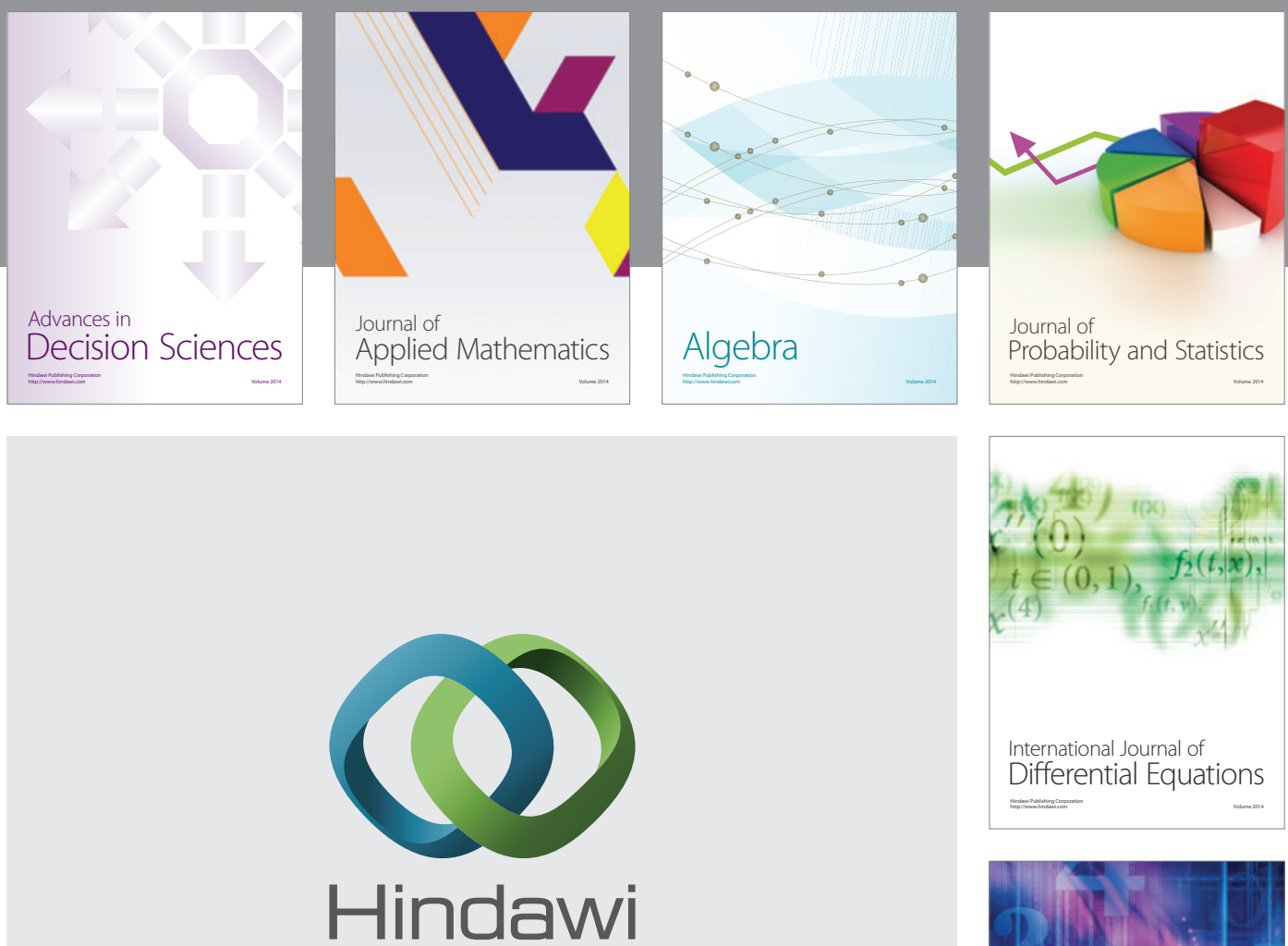

Submit your manuscripts at http://www.hindawi.com
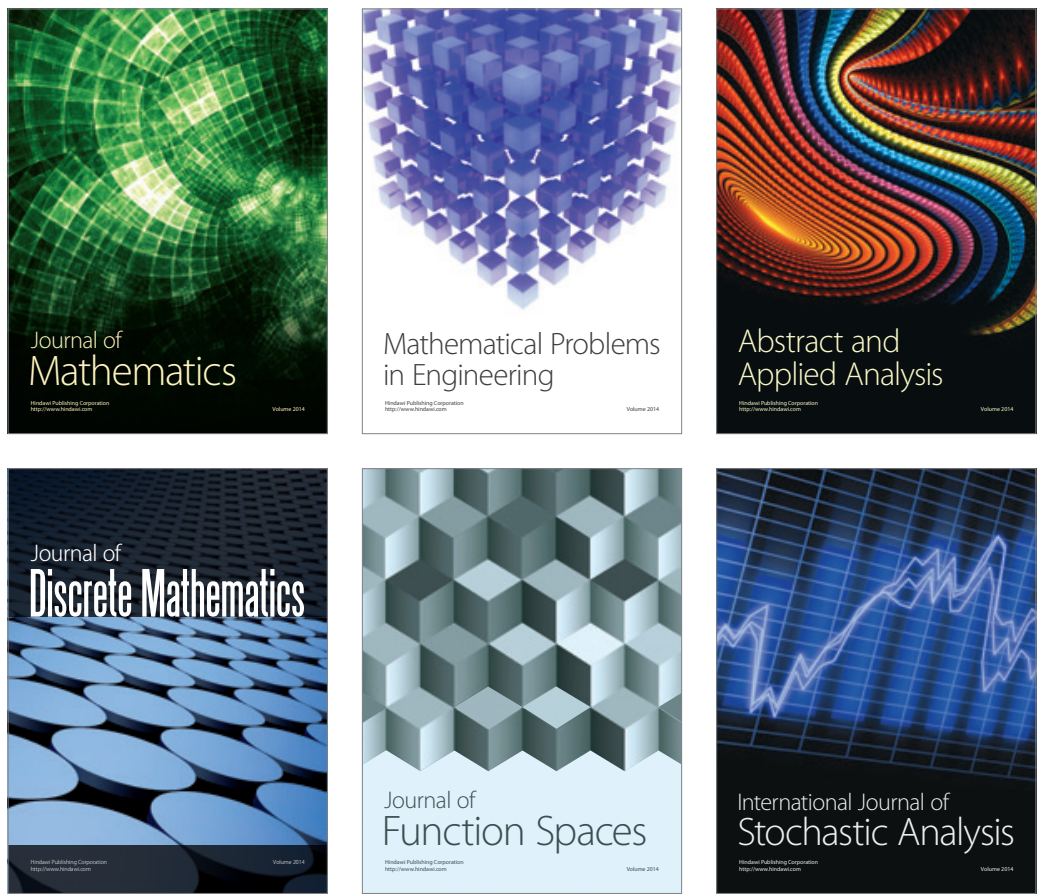

Journal of

Function Spaces

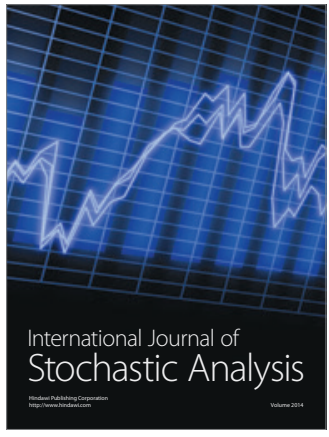

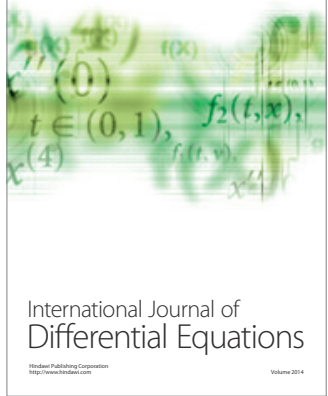
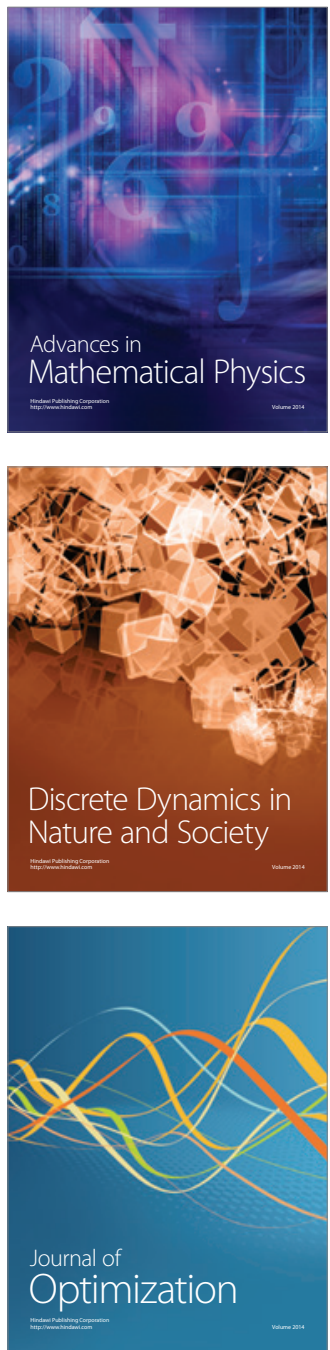